OPEN ACCESS

Edited by:

Kenneth Pfarr

Universitätsklinikum Bonn, Germany

Reviewed by:

Riti Sharan,

Texas A\&M Health Science Center,

United States

Rachel L. Roper,

Brody School of Medicine at East

Carolina University, United States

${ }^{*}$ Correspondence:

Lei Zhao

leizhao@hust.edu.cn

${ }^{\dagger}$ These authors have contributed equally to this work

Received: 30 June 2017 Accepted: 28 September 2017 Published: 18 October 2017

Citation:

$L i Y-Q$, Chen $Y-F$, Dang $Y-P$, Wang $Y$, Shang Z-Z, Ma Q, Wang Y-J, Zhang J, Luo L, Li Q-Q and Zhao L (2017)

Corilagin Counteracts IL-13Ra 1 Signaling Pathway in Macrophages to Mitigate Schistosome Egg-Induced Hepatic Fibrosis.

Front. Cell. Infect. Microbiol. 7:443 doi: 10.3389/fcimb.2017.00443

\section{Corilagin Counteracts IL-13R $\alpha 1$ Signaling Pathway in Macrophages to Mitigate Schistosome Egg-Induced Hepatic Fibrosis}

\author{
Yi-Qing $\mathrm{Li}^{1+}$, Yun-Fei Chen ${ }^{1+}$, Yi-Ping Dang ${ }^{1+}$, Yao Wang ${ }^{2}$, Zhen-Zhong Shang ${ }^{3}$, Qian $\mathrm{Ma}^{4}$, \\ Yu-Jie Wang ${ }^{1}$, Juan Zhang ${ }^{2}$, Lei Luo ${ }^{2}$, Quan-Qiang Li ${ }^{5}$ and Lei Zhao ${ }^{6 *}$ \\ ' Department of Vascular Surgery, Union Hospital, Tongji Medical College, Huazhong University of Science and Technology, \\ Wuhan, China, ${ }^{2}$ School of Clinical Medicine, Hubei University of Chinese Medicine, Wuhan, China, ${ }^{3}$ School of Basic Medical \\ Sciences, Guangxi University of Chinese Medicine, Nanning, China, ${ }^{4}$ School of Life Science, Hubei University, Wuhan, China, \\ ${ }^{5}$ School of Clinical Medicine, Guangxi University of Chinese Medicine, Nanning, China, ${ }^{6}$ Department of Infectious Diseases, \\ Union Hospital, Tongji Medical College, Huazhong University of Science and Technology, Wuhan, China
}

The IL-13R $\alpha 1$ signaling pathway and M2 macrophages play crucial roles in schistosome egg-induced hepatic fibrosis via the expression of pro-fibrotic molecules. This study aims to investigate the inhibitory effect and mechanism of action of corilagin on schistosome egg-induced hepatic fibrosis via the IL-13R $\alpha 1$ signaling pathway in M2 macrophages in vitro and in vivo. The mRNA and protein expression of IL-13R 1 1, PPAR $\gamma, \mathrm{KLF} 4$, SOCS1, STAT6, p-STAT6, and TGF- $\beta$ was measured in vitro with corilagin treatment after IL-13 stimulation and in vivo corilagin treatment after effectively killing the adult schistosomes in schistosome-infected mice. Histological analysis of liver tissue was assessed for the degree of hepatic fibrosis. The results revealed that corilagin significantly reduced the expression of PPAR $\gamma$, KLF4, SOCS1, p-STAT6, and TGF- $\beta$ compared with model group and praziquantel administration $(p<0.01$ or $p<0.05)$ in vivo and in vitro, which indicated a strong inhibitory effect of corilagin on IL-13R $\alpha 1$ signaling pathway. As well, the inhibitory effect of corilagin showed a significant dose-dependence $(p<0.05)$. The area of fibrosis and distribution of M2 macrophages in mouse liver tissue were reduced significantly and dose-dependently with corilagin treatment compared to model group or praziquantel administration ( $p<0.01$ or $p<0.05$ ), indicating that corilagin suppressed IL-13R $\alpha 1$ signaling pathway and $\mathrm{M} 2$ macrophage polarization effectively in vivo. Furthermore, the anti-fibrogenic effect persisted even when IL-13R $\alpha 1$ was up- or down-regulated in vitro. In conclusion, corilagin can suppress schistosome egg-induced hepatic fibrosis via inhibition of M2 macrophage polarization in the IL-13R $\alpha 1$ signaling pathway.

Keywords: corilagin, liver fibrosis, schistosome egg, IL-13R $\alpha 1$, M2 macrophage

\section{INTRODUCTION}

Schistosomiasis is an ancient parasitic disease that imposes a considerable economic and health burden, disabling more than it kills. As of 2014, over 207 million people were infected with schistosoma, and approximately 700 million people were at risk of infection (Shaker et al., 2014). Schistosomiasis is endemic in China, and China is one of the primary epidemic areas of Schistosoma 
japonicum (Colley et al., 2014; Song and Wu, 2015). Schistosomiasis hepatic fibrosis is a chronic immune disease caused by schistosome eggs that can lead to hepatosplenomegaly, ascites, portal hypertension and sometimes death as a result of upper gastrointestinal hemorrhage or hepatic encephalopathy.

Fibrosis is a component of normal tissue repair after acute inflammatory injury. However, it causes irreversible tissue damage in the case of chronic inflammation. Schistosomiasis hepatic fibrosis results from the soluble egg antigen (SEA) secreted by mature schistosome eggs, which induces the alternative activation of macrophages (M2 macrophages) that play a vital role in fibrogenesis (Martinez et al., 2009). In the acute phase, M2 macrophages can reduce the production of proinflammatory cytokines and prevent the induction of acute inflammatory lesions by eggs (Herbert et al., 2004). However, in the chronic phase, M2 macrophages secrete profibrotic cytokines, leading to hepatic fibrosis (Burke et al., 2009). Liver macrophages (Kupffer cells) are the major contributors to schistosome granuloma (Xie et al., 2015). After the SEA is released, the host immune response is polarized into a Th2biased response, with increased expression of IL-13 (Pearce and MacDonald, 2002). IL-13 was demonstrated to be detrimental in schistosomiasis (Fallon et al., 2000). And it is a key cytokine which strongly induced the expression of pro-fibrotic cytokines and M2 macrophage polarization via the IL-13 $\alpha 1$ signaling pathway (Liu et al., 2012; Sica and Mantovani, 2012; Song and $\mathrm{Wu}, 2015)$. IL-13 binding the IL-4R $\alpha / \mathrm{IL}-13 \mathrm{R} \alpha 1$ receptor complex triggers signal transduction and increases the expression of PPAR $\gamma, \mathrm{KLF} 4$ and SOCS1 (Biswas et al., 2012; Sica and Mantovani, 2012). These M2 cytokines stimulate the Kupffer cells and hepatic stellar cells to produce TGF- $\beta$, a crucial factor in fibrosis, and accelerate fibrosis progression (Fabregat et al., 2016). Thus, inhibition of M2 polarization of macrophages and IL-13R $\alpha 1$ signaling pathway may be the key to suppressing schistosomiasis hepatic fibrosis.

Currently, the most widely used drug for the treatment of schistosomiasis is praziquantel, which can kill the mature parasite but has little effect on schistosome eggs (Gryseels et al., 2006). Studies have shown that schistosomiasis hepatic fibrosis and granulomatous inflammatory disease continue even after effective anti-Schistosoma treatment (Cioli and Pica-Mattoccia, 2003; Southgate et al., 2005; Gryseels et al., 2006). Resistance to praziquantel has also been reported (Ross et al., 2002; Burke et al., 2009). Therefore, suppressing fibrosis and granuloma formation after effective killing of mature Schistosoma has become a key

\footnotetext{
Abbreviations: ANOVA, Analysis of variance; ELISA, Enzyme-Linked Immunosorbent Assay; FBS, fetal bovine serum; HE staining, Haematoxylin and eosin staining; HSC, Hepatic stellate cell; HSV1, Herpes simplex virus 1; IL-4R $\alpha$, Interleukin-4 receptor alpha; IL-13, Interleukin-13; IL-13R $\alpha$, Interleukin-13 receptor alpha 1; KLF4, Krüppel-like factor4; MOI, Multiplicity of infection; mRNA, Messenger ribonucleic acid; NF- $\kappa$, Nuclear factor kappalight-chain-enhancer of activated B cells; PBS, Phosphate buffer saline; PPAR $\gamma$, Peroxisome proliferator-activated receptor gamma; p-STAT6, Phosphorylated signal transducer and activator of transcription 6; qPCR, Quantitative polymerase chain reaction; RPMI medium, Roswell Park Memorial Institute medium; SD, Standard deviation; SEA, Soluble egg antigen; siRNA, Small interfering ribonucleic acid; SOCS1, Suppressor of cytokine signaling 1; STAT6, Signal transducer and activator of transcription 6; TGF- $\beta$, Transforming growth factor beta; TNF- $\alpha$, tumor necrosis factor alpha; WB, Western blot.
}

issue in the treatment of chronic and advanced schistosomiasis (Chu et al., 2011). Many researchers have tried to find new therapeutics to inhibit schistosomiasis hepatic fibrosis.

Corilagin $\left(\mathrm{C}_{27} \mathrm{H}_{22} \mathrm{O}_{18}\right)$ is an active component of many medicinal plants, with a variety of pharmacological activities including anti-tumor, anti-oxidative, anti-atherogenic, thrombolytic, anti-hypertensive, hepatoprotective, antiviral, antibacterial and anti-inflammatory effects (Zhao et al., 2008; Guo et al., 2010, 2015a; Jin et al., 2013; Li et al., 2017). Its known chemical structure was presented in our previously published paper (Zhao et al., 2008).

We previously confirmed the anti-fibrogenic effect of corilagin (Huang et al., 2013; Yang et al., 2016) and investigated intervention with the IL-13R signaling pathway in HSC (Li et al., 2016) and a mouse model without praziquantel treatment (Du et al., 2016; Yang et al., 2016). In this study, IL-13 was used in vitro to stimulate Ana-1 cells to simulate egg-induced fibrosis. For the in vivo study, praziquantel was administered after the mice were infected by the cercaria to kill the adult schistosomes. We investigated the inhibitory effect of corilagin on the molecules involved in IL-13R $\alpha 1$ signaling pathway (PPAR $\gamma, \mathrm{KLF} 4$, SOCS1 and p-STAT6) and egg-induced fibrogenesis (TGF- $\beta$ ) in vitro and in vivo. Furthermore, we observed the fibrosis degree and distribution of M2 macrophages in schistosomiasis mouse liver by the method of histology and immunohistochemistry in animal model. Through this study, we aimed to develop a new strategy for the treatment of schistosomiasis hepatic fibrosis which praziquantel had little effect on.

\section{MATERIALS AND METHODS}

Chemicals and reagents could be seen in the Supplementary Data Sheet 2.

\section{Cell Culture}

Ana- 1 cell line is a well-established murine macrophage cell line, and it can be transformed into either M1 or M2 macrophage with

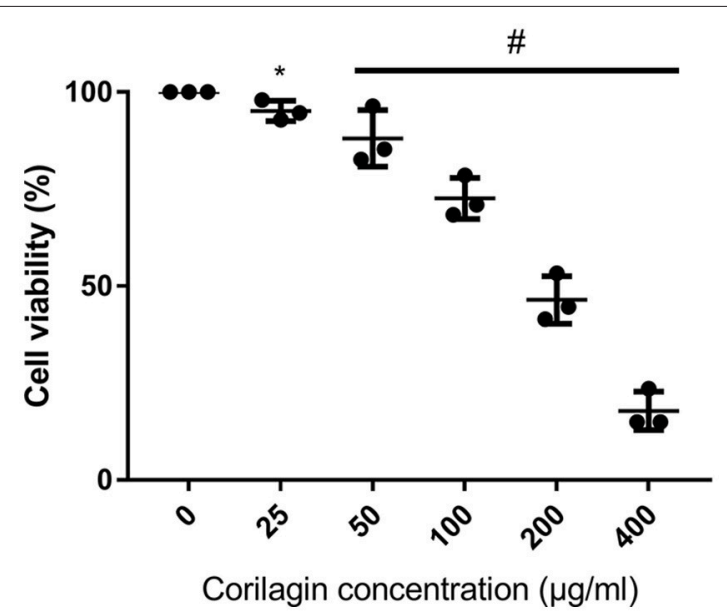

FIGURE 1 | CCK8 assay of Ana-1 cell viability after Corilagin treatment for $24 \mathrm{~h}$. $\# p<0.05$ determined by one-way ANOVA and significant differences from the respective values determined by S-N-K method $(n=3) .{ }^{*} p<0.05$ compared with the $0 \mu \mathrm{g} / \mathrm{ml}$ group ( $n=3$, student's $t$-test). 

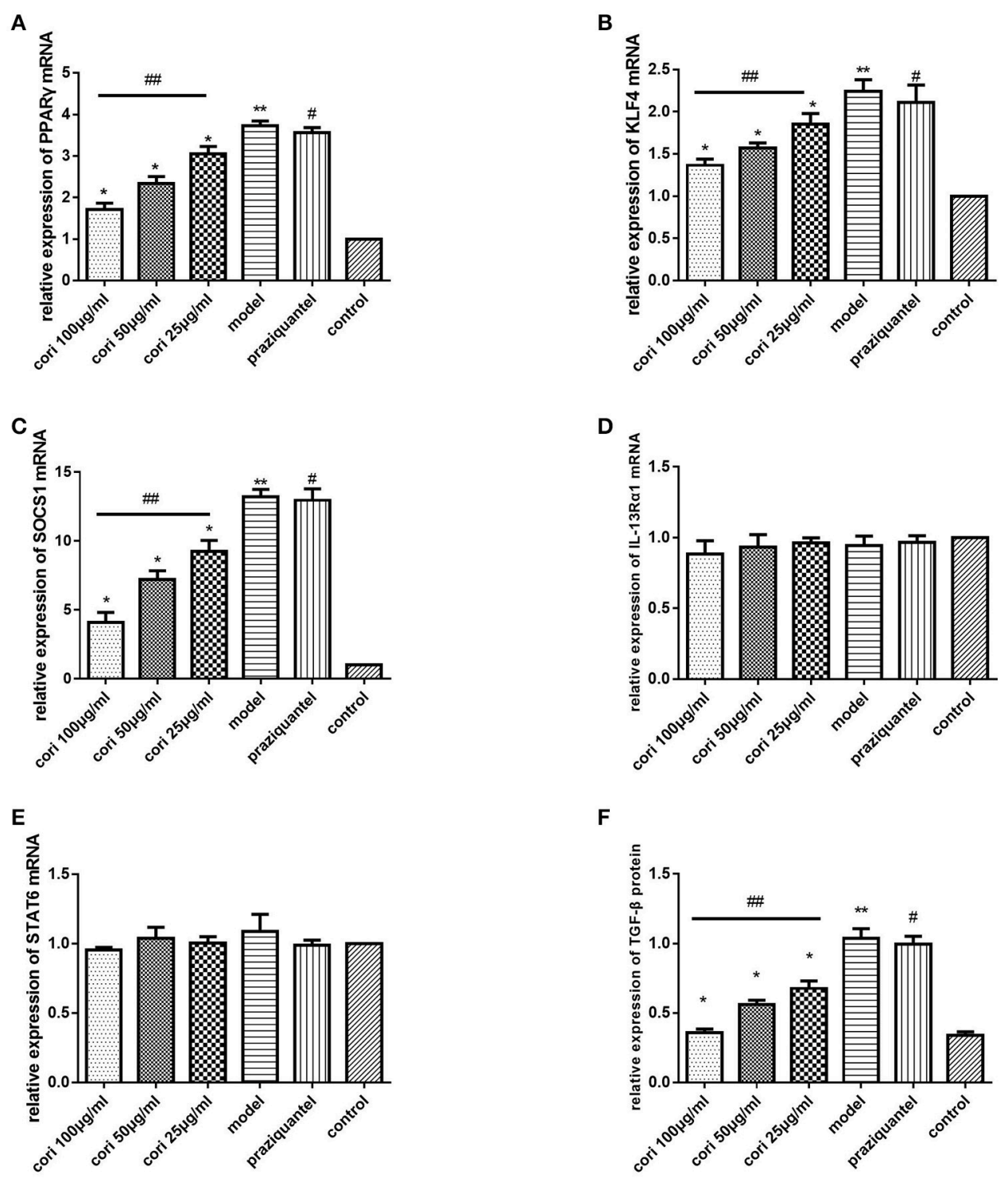

$\mathbf{F}$
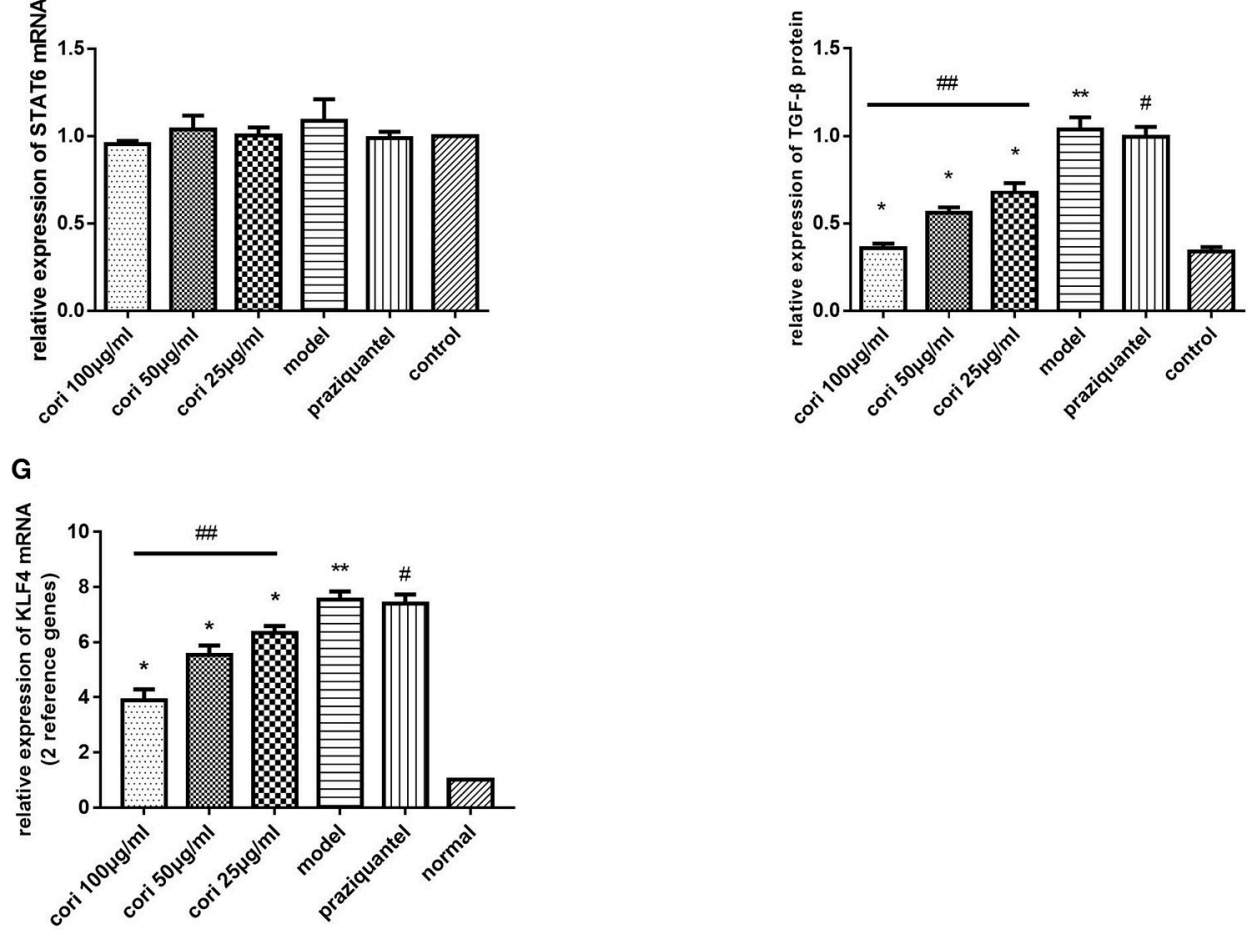

FIGURE 2 | Effect of Corilagin on mRNA expression of PPAR $\gamma$, KLF4, SOCS1, IL-13R $\alpha 1$, STAT6, and protein expression of TGF- $\beta$ after IL-13 stimulation in Ana-1 cells. Ana-1 cells were stimulated with rlL-13 for $24 \mathrm{~h}$ except control group. Subsequently, the cells were administrated with corilagin or praziquantel for $24 \mathrm{~h}$ respectively. Corilagin showed a dose-dependent inhibitory effect on IL-13R $\alpha 1$ signaling pathway after IL-13 stimulation. (A) The PPAR $\gamma$ mRNA expression levels detected by real-time qPCR. ${ }^{*} p<0.01$ compared with the model group $\left(n=3\right.$, student's $t$-test); ${ }^{* *} p<0.01$ compared with the control group ( $n=3$, student's $t$-test); $\# p<0.01$ compared with the control group $(n=3$, student's $t$-test); \#\#p < 0.05 determined by one-way ANOVA and significant differences from the respective values 


\section{FIGURE 2 | Continued}

determined by S-N-K method $(n=3)$. (B) The KLF4 mRNA expression levels detected by real-time qPCR. ${ }^{*} p<0.05$ compared with the model group $(n=3$, student's $t$-test); ${ }^{* *} p<0.01$ compared with the control group $(n=3$, student's $t$-test); $\# p<0.01$ compared with the control group $(n=3$, student's $t$-test); \#\#p $<0.05$ determined by one-way ANOVA and significant differences from the respective values determined by S-N-K method $(n=3)$. (C) The SOCS1 mRNA expression levels detected by real-time qPCR. ${ }^{*} p<0.01$ compared with the model group $\left(n=3\right.$, student's $t$-test); ${ }^{* *} p<0.01$ compared with the control group $(n=3$, student's $t$-test); $\# p<0.01$ compared with the control group ( $n=3$, student's $t$-test); \#\#p<0.05 determined by one-way ANOVA and significant differences from the respective values determined by S-N-K method $(n=3)$. (D) The IL-13R $\alpha 1$ mRNA expression levels detected by real-time qPCR. It was shown that no difference among all groups. (E) The STAT6 mRNA expression levels detected by real-time qPCR. It was shown that no difference among all groups. (F) Effect of Corilagin on TGF- $\beta$ protein expression detected by ELISA. ${ }^{*} p<0.01$ compared with the model group $\left(n=3\right.$, student's $t$-test); ${ }^{* *} p<0.01$ compared with the control group $(n=3$, student's $t$-test); $\# p<0.01$ compared with the control group $(n=3$, student's $t$-test); \#\#p < 0.05 determined by one-way ANOVA and significant differences from the respective values determined by S-N-K method $(n=3)$. (G) The KLF4 mRNA expression levels with two reference genes detected by real-time qPCR. ${ }^{*} p<0.05$ compared with the model group $\left(n=3\right.$, student's $t$-test); ${ }^{* *} p<0.01$ compared with the control group $(n=3$, student's $t$-test); \#p $<0.01$ compared with the control group ( $n=3$, student's $t$-test); \#\#p $<0.05$ determined by one-way ANOVA and significant differences from the respective values determined by S-N-K method $(n=3)$. Data are shown with mean with SD.

some administration (Chen et al., 2012). It was purchased from the Type Culture Collection of the Chinese Academy of Sciences, Shanghai, China. The cells were cultured in RPMI-1640 medium containing $10 \%$ fetal bovine serum in an incubator at $37^{\circ} \mathrm{C}, 5 \%$ $\mathrm{CO}_{2}$ and saturated humidity.

\section{Corilagin Cytotoxicity Assays}

According to the previously published paper (Li et al., 2016), we evaluated cell viability using the CCK8 assay. The Ana1 cells were plated at a density of $1 \times 10^{4} / \mathrm{mL}$ cells in $96-$ well culture plates with different concentrations of corilagin $(400,200,100,50$, and $25 \mathrm{mg} / \mathrm{mL})$. The Ana- 1 cells without corilagin administration was set as a positive control, and a blank well-containing the medium culture was set as a negative control. After $24 \mathrm{~h}$, the cell morphology was observed, and 10 $\mu \mathrm{l}$ of CCK-8 solution was added to each well, which was then incubated for $2 \mathrm{~h}$ before the absorbance was measured at $450 \mathrm{~nm}$ using a microplate reader. Three wells in each group were measured.

\section{In Vitro Corilagin Treatment after II-13 Stimulation}

Recombinant IL-13 was diluted to $5 \mu \mathrm{g} / \mathrm{ml}$ with RPMI-1640 medium containing $5 \%$ fetal bovine serum and stored at $-20^{\circ} \mathrm{C}$. Praziquantel was diluted to $5 \mathrm{pg} / \mathrm{ml}$ with $\mathrm{PBS}$ and stored at $4^{\circ} \mathrm{C}$. Corilagin was diluted to $2 \mathrm{mg} / \mathrm{ml}$ with $\mathrm{PBS}$ and stored at $4^{\circ} \mathrm{C}$. To explore the anti-fibrogenic effect of corilagin with the praziquantel intervention via the IL$13 \alpha 1$ signaling pathway, Ana-1 cells were divided into six groups. The high- $(100 \mu \mathrm{g} / \mathrm{ml})$, medium- $(50 \mu \mathrm{g} / \mathrm{ml})$, and lowconcentration $(25 \mu \mathrm{g} / \mathrm{ml})$ corilagin groups were stimulated with rIL-13 $(50 \mathrm{ng} / \mathrm{ml})$ for $24 \mathrm{~h}$, followed by treatment of corilagin for $24 \mathrm{~h}$, and no praziquantel was administered. The praziquantel group was stimulated with rIL-13 $(50 \mathrm{ng} / \mathrm{ml})$ for $24 \mathrm{~h}$, followed by treatment of praziquantel $(5 \mathrm{pg} / \mathrm{ml})$ for $24 \mathrm{~h}$. The model group was incubated with rIL-13 (50 ng/ml) only. The untreated group was the control group.

\section{IL-13R $\alpha 1$ Up-Regulation by Lentivirus Transfection}

IL-13R $\alpha 1$ was up-regulated by means of lentivirus transfection containing pre-IL-13R $\alpha 1$ and green fluorescent protein. Ana1 cells were cultured in enhanced infection solution with the vehicle for $96 \mathrm{~h}$ at a MOI of 40 . The transfection efficiency was observed under a fluorescence microscope (Olymbus IX2 series microscope). To further confirm the effect of corilagin on the IL-13 $\alpha 1$ pathway, Ana-1 cells were divided into seven groups for an up-regulation experiment. The group stimulated with rIL-13 alone without IL-13R $\alpha 1$ up-regulation was included in the previous experiments. Therefore, we set up this part of the experiment without the group of rIL-13 stimulation alone. In the high- $(100 \mu \mathrm{g} / \mathrm{ml})$, medium- $(50 \mu \mathrm{g} / \mathrm{ml})$, lowconcentration $(25 \mu \mathrm{g} / \mathrm{ml})$ corilagin up-regulation groups, the IL$13 \mathrm{R} \alpha 1$ up-regulated Ana-1 cells were stimulated with rIL-13 $(50 \mathrm{ng} / \mathrm{ml})$ for $24 \mathrm{~h}$ before corilagin was added. In the model upregulation group, the IL-13R $\alpha 1$ up-regulated Ana-1 cells were incubated with $\mathrm{rIL}-13(50 \mathrm{ng} / \mathrm{ml})$ only. In the up-regulation group, the IL-13R $\alpha 1$ up-regulated Ana-1 cells were cultured without any treatment. In the empty-vector group, the Ana1 cells were cultured in enhanced infection solution with the empty vector for $96 \mathrm{~h}$ only. The untreated group was the control group.

\section{IL-13R $\alpha 1$ Down-Regulation by siRNA Transfection}

IL-13R $\alpha 1$ was down-regulated by means of siRNA transfection. The siRNA was purchased from RiboBio company (Guangzhou, China), and the specificity of siRNA was checked by Primer BLAST. The target sequence of the siRNA was CCTGCACTGGAAGAAGTAT, and the concentration of the siRNA was $0.02 \mathrm{nmol} / \mu \mathrm{l}$. The siRNA contained cy3 dye. Ana- 1 cells were incubated in RPMI-1640 medium containing $5 \%$ fetal bovine serum with $0.25 \%$ (5 $\mu$ l Lipofectamine ${ }^{\mathrm{TM}} 2000$ in $2 \mathrm{ml}$ cell culture medium) Lipofectamine ${ }^{\mathrm{TM}} 2000$ (purchased from Invitrogen) and 100 pmol of siRNA for $6 \mathrm{~h}$, then cultured with RPMI-1640 medium containing 5\% fetal bovine serum that did not contain siRNA or Lipofectamine ${ }^{\mathrm{TM}} 2000$ for $48 \mathrm{~h}$. Subsequently, the transfection efficiency was observed under a fluorescence microscope. To further confirm the effect of corilagin on the IL-13 $\alpha 1$ pathway, Ana-1 cells were divided into seven groups in the down-regulation experiment. In the high- $(100 \mu \mathrm{g} / \mathrm{ml})$, medium- $(50 \mu \mathrm{g} / \mathrm{ml})$, low-concentration $(25 \mu \mathrm{g} / \mathrm{ml})$ corilagin down-regulation groups, the IL-13R $\alpha 1$ down-regulated Ana-1 cells were stimulated with rIL-13 $(50 \mathrm{ng} / \mathrm{ml})$ for $24 \mathrm{~h}$ before corilagin was added. In the model 
A

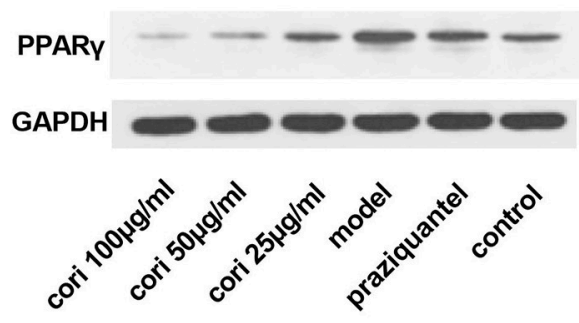

B

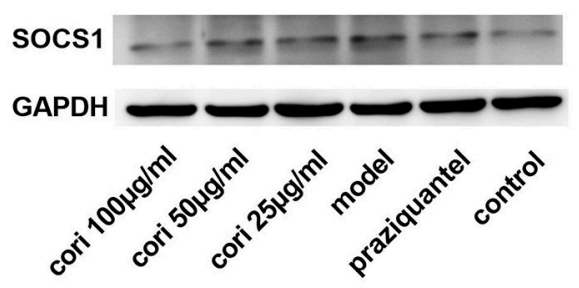

C

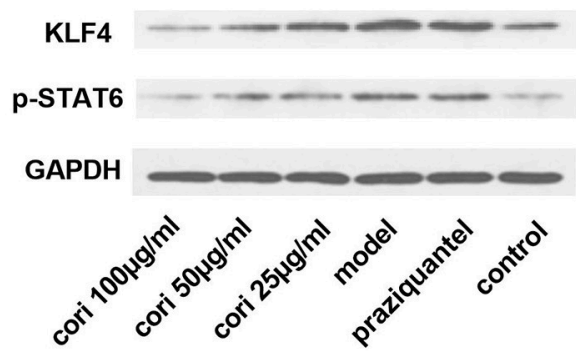

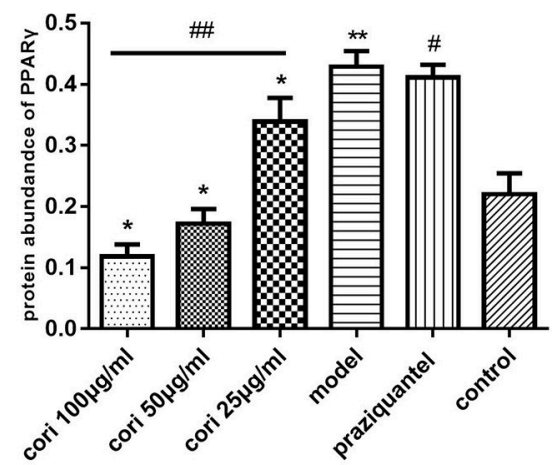
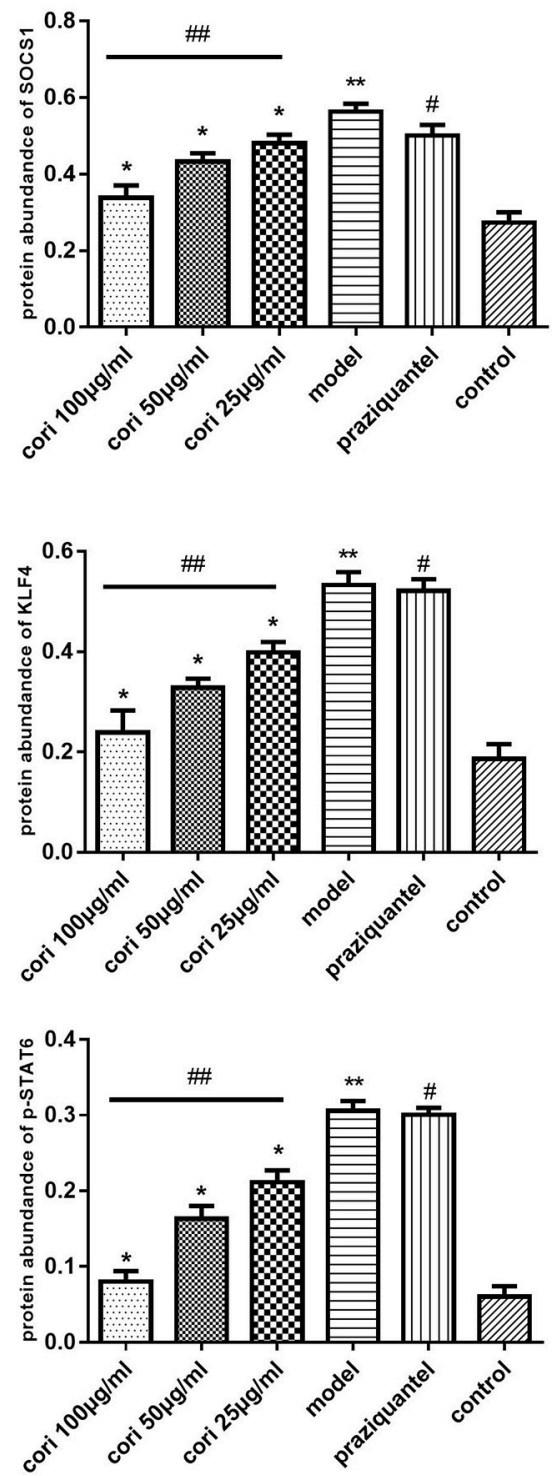

FIGURE 3 | Effect of Corilagin on protein abundance of PPAR $\gamma$, KLF4, SOCS1, and p-STAT6 after IL-13 stimulation in Ana-1 cells. Ana-1 cells were stimulated with rlL-13 for $24 \mathrm{~h}$ except control group. Subsequently, the cells were administrated with corilagin or praziquantel for $24 \mathrm{~h}$ respectively. Corilagin showed a

dose-dependent inhibitory effect on $\mathrm{IL}-13 \mathrm{R} \alpha 1$ signaling pathway after $\mathrm{L}-13$ stimulation. (A) The PPAR $\gamma$ protein abundance detected by western blot assay. ${ }^{*} p<0.01$ 
FIGURE 3 | Continued

compared with the model group ( $n=3$, student's $t$-test); ** $p<0.01$ compared with the control group $(n=3$, student's $t$-test); \#p $<0.01$ compared with the control group ( $n=3$, student's $t$-test); \#\#p < 0.05 determined by one-way ANOVA and significant differences from the respective values determined by S-N-K method $(n=3)$. (B) The SOCS1 protein abundance detected by western blot assay. ${ }^{*} p<0.01$ compared with the model group $\left(n=3\right.$, student's $t$-test); ${ }^{* *} p<0.01$ compared with the control group ( $n=3$, student's $t$-test); \#p $<0.01$ compared with the control group ( $n=3$, student's $t$-test); \#\#p $<0.05$ determined by one-way ANOVA and significant differences from the respective values determined by S-N-K method $(n=3)$. (C) The KLF4 and p-STAT6 protein abundance detected by western blot assay. ${ }^{*} p<0.01$ compared with the model group $\left(n=3\right.$, student's $t$-test); ${ }^{* *} p<0.01$ compared with the control group $(n=3$, student's $t$-test); \#p $<0.01$ compared with the control group ( $n=3$, student's $t$-test); \#\# $p<0.05$ determined by one-way ANOVA and significant differences from the respective values determined by $\mathrm{S}-\mathrm{N}-\mathrm{K}$ method $(n=3)$. Data are shown with mean with SD.

down-regulation group, the IL-13R $\alpha 1$ down-regulated Ana-1 cells were incubated with rIL-13 $(50 \mathrm{ng} / \mathrm{ml})$ only and cultured without any treatment. In the empty-vector group, the Ana-1 cells were transfected by the empty vector. The untreated group was the control group.

\section{Corilagin Treatment on Mouse Schistosomiasis Model after Praziquantel Administration}

The animal experiments were approved by the Tongji Medical College, HUST Institutional Animal Care and Use Committee ([2016] IACUC Number: 598). A total of 42 male C57BL/6 mice, 6 to 8 weeks old and weighing 18-22 g, were purchased from the Hubei Provincial Center for Disease Control and Prevention (Wuhan, China). The mice were randomized into seven groups, with six mice per group. The mice were fed an untreated diet and given access to water under standard laboratory conditions at a temperature of $25 \pm 2{ }^{\circ} \mathrm{C}$, a relative humidity of $50 \pm 15 \%$ and a normal circadian rhythm (12-h dark/12-h light). All study protocols were conducted in accordance with the Guidelines for the Care and Use of Laboratory Animals of Huazhong University of Science and Technology and approved by the Ethics Committee of Union Hospital, Tongji Medical College, Huazhong University of Science and Technology. The control group served as a blank control without any administration or infection. The animals in all groups except the control group were infected with $25 \pm 5$ Schistosoma japonicum cercaria via the abdominalpatch method (Huang et al., 2013). After anaesthesia with 2\% pentobarbital sodium (intraperitoneal injection, $45 \mathrm{mg} / \mathrm{kg}$ ), the abdominal skin without hair was exposed to the cercaria carried by Oncomelania hupensis for $30 \mathrm{~min}$ to infect the mice. Four weeks after infection, the animals in all groups except the control group were administered praziquantel orally at $500 \mathrm{mg} / \mathrm{kg} \cdot \mathrm{d}$ for 5 days to kill the adult schistosomes. Subsequently, the high(80 $\mathrm{mg} / \mathrm{kg} \cdot \mathrm{d})$, medium- $(40 \mathrm{mg} / \mathrm{kg} \cdot \mathrm{d})$, and low-concentration $(20 \mathrm{mg} / \mathrm{kg} \cdot \mathrm{d})$ corilagin groups were administered corilagin orally for 21 days. The praziquantel group as a positive control continued to be administered praziquantel orally at $500 \mathrm{mg} / \mathrm{kg} \cdot \mathrm{d}$ for 21 days after the initial 5 days of praziquantel treatment. The levofloxacin group as a negative control was administered levofloxacin orally at $100 \mathrm{mg} / \mathrm{kg} \cdot \mathrm{d}$ for 21 days. The model infection group was administered with no more drugs after 5day praziquantel treatment. Finally, the mice were euthanized after anesthetization of $4 \%$ chloral hydrate by intraperitoneal injection and the specimens were collected. Blood was collected via retro-orbital route, centrifuged at 3,500 rpm for $10 \mathrm{~min}$ and sera were stored at $-20^{\circ} \mathrm{C}$ for ELISA. Part of the liver tissue was fixed in $10 \%$ formalin for tissue examination. The rest was stored in liquid nitrogen for PCR and Western-blot assays. During the dissection of mice, the adult schistosomes were not observed in the liver of mice or in the portal system, which indicated the 5-day praziquantel treatment was effective on killing the adult schistosomes.

\section{Real-Time Quantitative PCR}

The expression of PPAR $\gamma$, KLF4, SOCS1 and STAT6 was quantified by real-time qPCR. The operation and analysis was performed according to our previously published protocol (Guo et al., 2015b). Total RNA in Ana-1 cells was isolated using RNAiso Plus (TaKaRa Company). The A260/280 ratio of the mRNA was detected by spectrophotometer (UV751GD UV/VIS spectrophotometer, Implen company, German), and only the samples whose A260/280 ratios were between 1.8 and 2.2 were used. The cDNAs were produced with the PrimeScript ${ }^{\mathrm{TM}}$ RT reagent kit (TaKaRa company) and incubated at $37^{\circ} \mathrm{C}$ for $15 \mathrm{~min}$ and $85^{\circ} \mathrm{C}$ for $5 \mathrm{~s}$. Real-time PCR reactions were performed using a StepOne Plus device (Applied Biosystems) at $95^{\circ} \mathrm{C}$ for $10 \mathrm{~s}$, followed by 40 cycles of $95^{\circ} \mathrm{C}$ for $5 \mathrm{~s}$ and $60^{\circ} \mathrm{C}$ for $20 \mathrm{~s}$ according to the instructions for the SYBR Premix Ex Taq kit (TaKaRa company). The standard curves indicated that all the slopes ranged from -3.3515 to -3.222 and the $R^{2}$ values were greater than 0.99 . Therefore, the data were analyzed by the $2^{-\triangle \Delta C T}$ method. The number of PCR cycles varied according to the expression level of the target gene. An appropriate primer concentration and number of cycles was determined to ensure that the PCR was taking place in the linear range and thereby guaranteed a proportional relationship between input RNA and the cycles readout. All primers were synthesized by TSINGKE (Wuhan, China). GAPDH and $\beta$-actin were used as reference genes. In our study, the trends of KLF4 expression were consistent in the case of two reference genes (GAPDH and $\beta$ actin). Therefore, we chose GAPDH as the reference gene for the following qPCR assay. Three sets of duplicate data were included for statistical analysis. The primers were constructed and purchased from Tsingke Biological Technology Company (Beijing, China). The specificity of primer was checked by Primer Blast. The standard curves and amplification curves could be seen in Supplementary Data Sheet 1.

\section{Western-Blot Analysis}

The abundance of PPAR $\gamma$, KLF4, SOCS1, STAT6 and p-STAT6 was detected by Western blot. The operation and analysis 


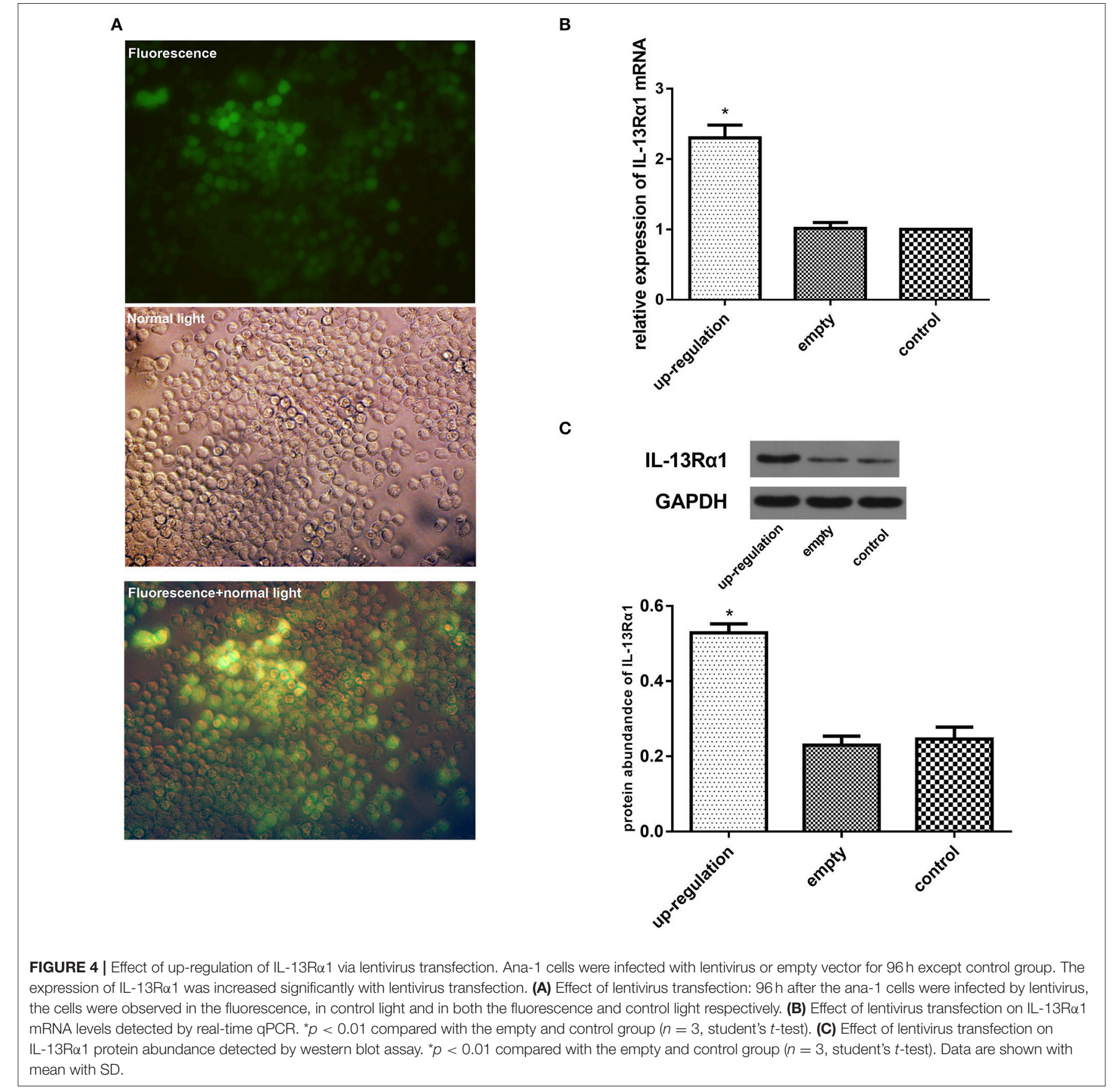

B

were performed as described in a previously published paper (Wang et al., 2017). The protein was separated on SDS-PAGE gels and then transferred to nitrocellulose filter membrane, which was blocked overnight with $5 \%$ non-fat milk in TBST. Subsequently, the membrane was probed with the indicated antibody at $4^{\circ} \mathrm{C}$ before being washed three times in TBST and then incubated with an HRP-labeled secondary antibody. The dilutions of the primary antibodies were as follows: PPAR $\gamma$ (Proteintech Company, Wuhan, China), 1:1,000; KLF4 (Santa Cruz Biotechnology Company, Dallas, TX, US), 1:300; SOCS1
(Abclonal Technology, Woburn, MA, US), 1:500; p-STAT6 (CST, Boston, MA, US), 1:1,000.

\section{Elisa Assay}

The TGF- $\beta$ levels in the cell supernatant or in the sera of the mice were determined by ELISA. The sera were assayed for TGF- $\beta$ with Mouse TGF- $\beta$ ELISA kits (Elabscience, Wuhan, China). The procedures were conducted according to the instruction manuals for the kits. Sterile PBS was used as control. 
A
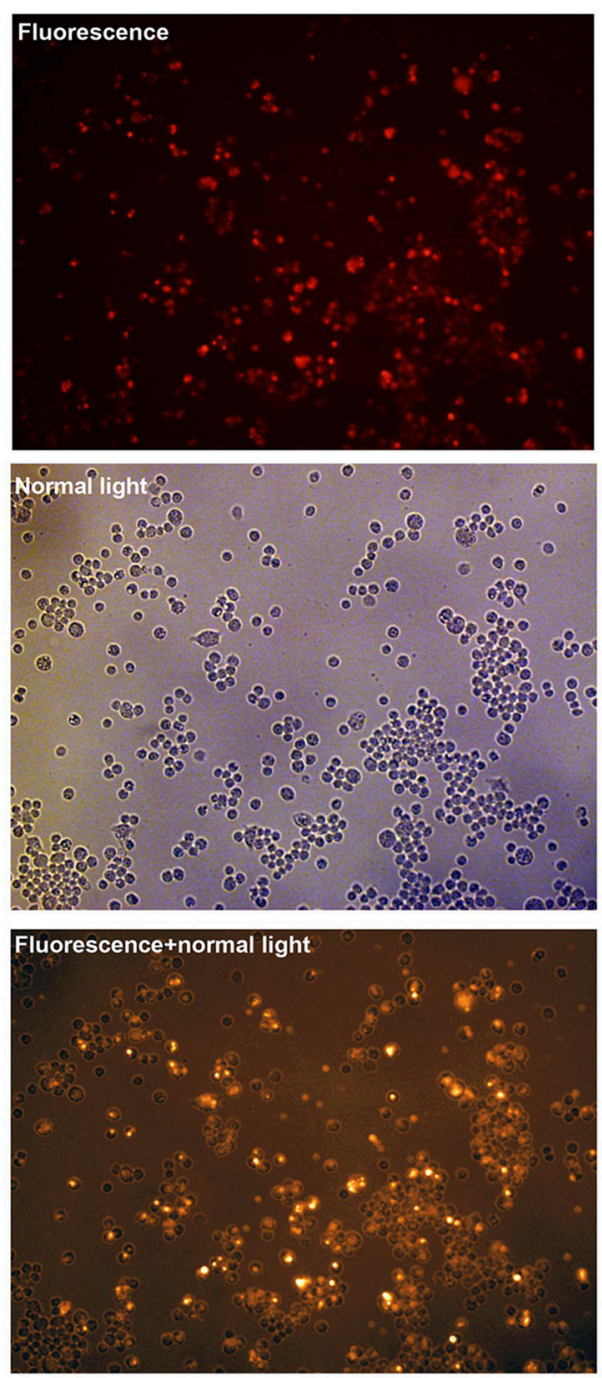

B

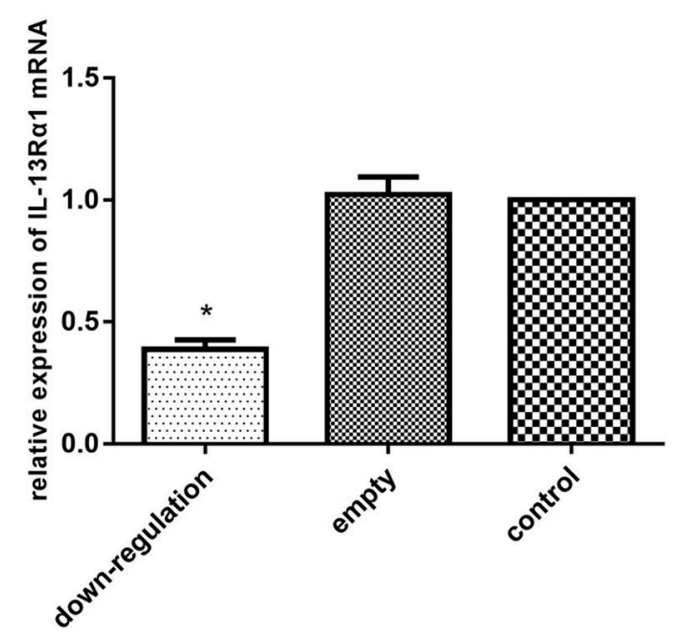

C

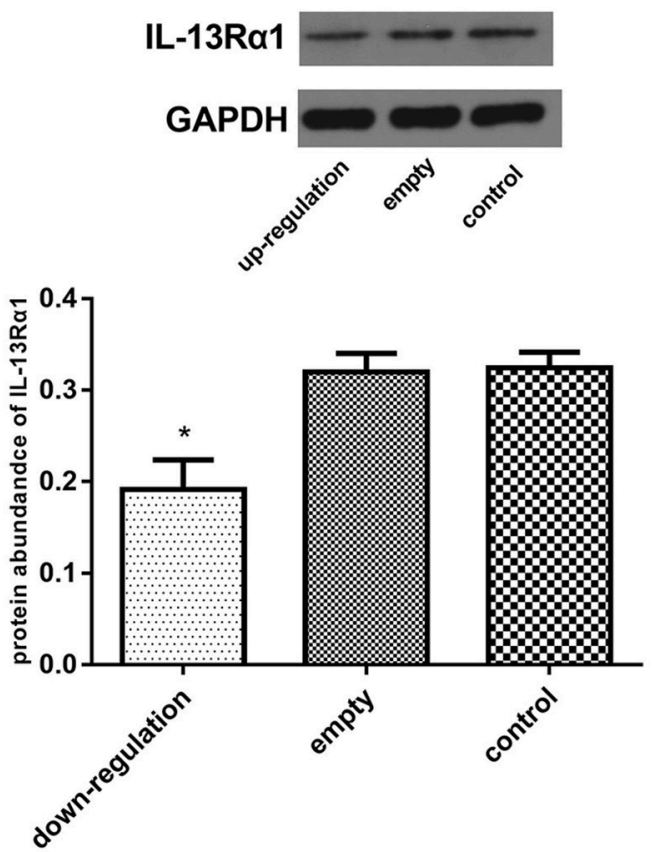

FIGURE 5 | Effect of down-regulation of IL-13Ra1 via siRNA transfection. The expression of IL-13Ra 1 was decreased significantly with siRNA transfection. Ana-1 cells were infected with IL-13R $\alpha 1$ siRNA or empty vector for $48 \mathrm{~h}$ except control group. (A) Effect of siRNA transfection: $48 \mathrm{~h}$ after the ana-1 cells were infected, the cells were observed in the fluorescence, in control light and in both the fluorescence and control light respectively. (B) Effect of siRNA transfection on IL-13R 1 mRNA levels detected by real-time qPCR. ${ }^{*} p<0.01$ compared with the empty and control group ( $n=3$, student's $t$-test). (C) Effect of siRNA transfection on IL-13R 1 protein abundance detected by western blot assay. ${ }^{*} p<0.01$ compared with the empty and control group $(n=3$, student's $t$-test). Data are shown with mean with SD.

\section{Histological Analysis}

Hepatic tissue samples were obtained, formalin-fixed and paraffin-embedded. As in our previously published paper, haematoxylin and eosin (HE) staining (Jin et al., 2015) and Masson's trichrome staining (Li et al., 2016) were performed. Masson's trichrome stains hepatic cells in red and collagen in blue, which could help to clarify the degree of fibrosis. CD206 was a cell surface marker which could distinguish M2 macrophages from M1 macrophages.
Immunohistochemistry for CD206 was performed to observe the M2 macrophages distribution in the liver tissue sections. The operation and analysis was performed according to our previously published protocol (Du et al., 2016). CD206 antibody was purchased from Proteintech Group, INC (Chicago, USA), catalog number 18704-1-AP, and its dilution was 1:200. The dilution of CD206 antibody was 1:200. The granuloma area and IOD of CD206 was measured by Imag-Pro Plus 6.0. 

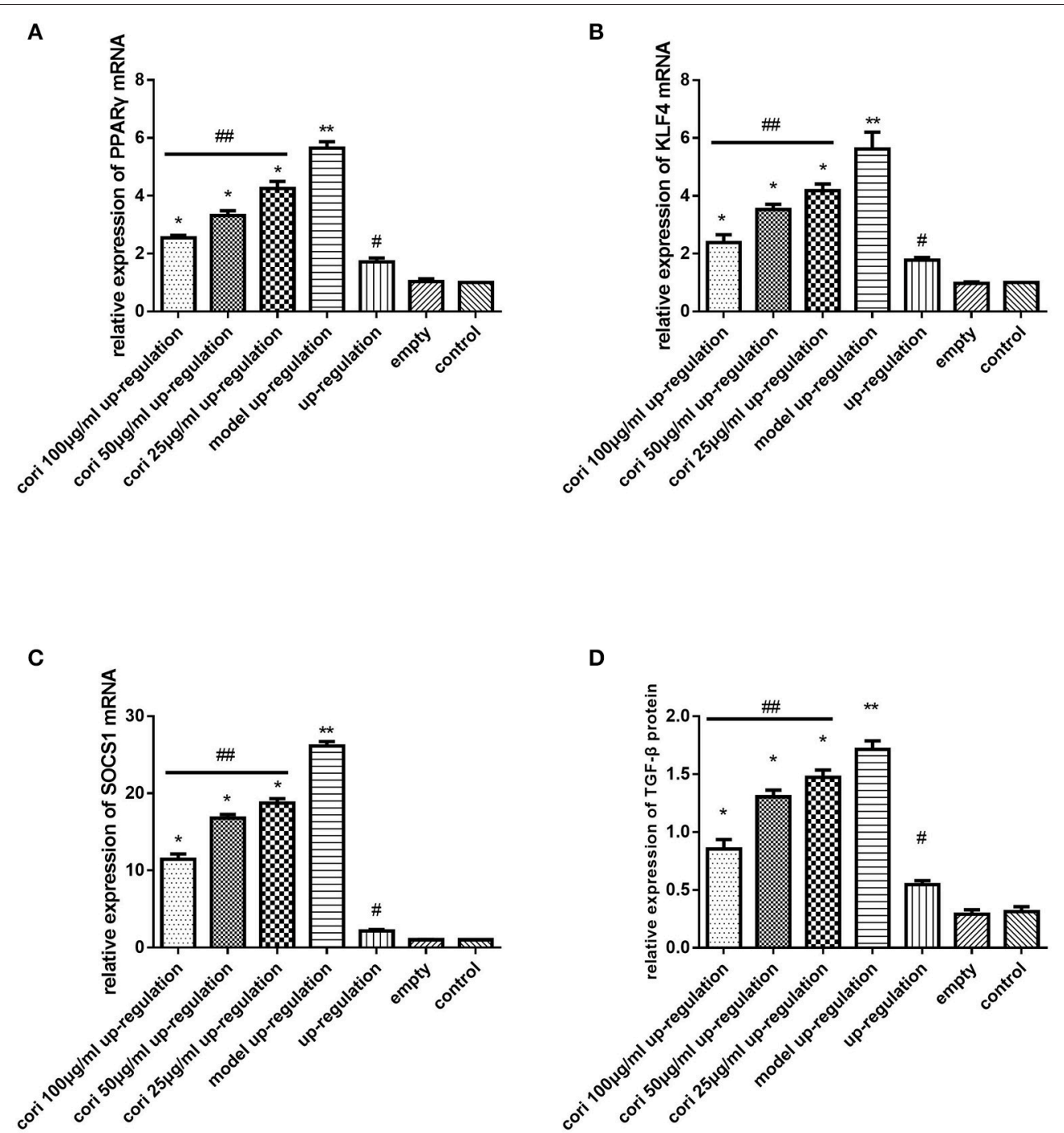

FIGURE 6 | Effect of Corilagin on mRNA expression of PPAR $\gamma$, KLF4, SOCS1, IL-13R $\alpha$ 1, STAT6 and protein expression of TGF- $\beta$ after IL-13 stimulation in IL-13R 1 up-regulated Ana-1 cells. Ana-1 cells was infected with IL-13R $\alpha 1$ lentivirus or empty vector for $96 \mathrm{~h}$ except control group. Subsequently, the corilagin groups and model up-regulation group were administrated with $\mathrm{rlL}-13$ for $24 \mathrm{~h}$. Then the corilagin groups were treated with corilagin of different concentrations. Real-time qPCR and ELISA were performed. The dose-dependent anti-fibrogenesis effect of corilagin still persisted when IL-13Ra1 was up-regulated. (A) The PPAR $\gamma$ mRNA expression levels detected by real-time qPCR. ${ }^{*} p<0.01$ compared with the model up-regulation group $\left(n=3\right.$, student's $t$-test); ${ }^{* *} p<0.01$ compared with the up-regulation group ( $n=3$, student's $t$-test); \#p $<0.01$ compared with the empty and control group $(n=3$, student's $t$-test); \#\# $p<0.05$ determined by one-way ANOVA and significant differences from the respective values determined by S-N-K method $(n=3)$. (B) The KLF4 mRNA expression levels detected by real-time qPCR. ${ }^{*} p<0.01$ compared with the model up-regulation group $\left(n=3\right.$, student's $t$-test); ${ }^{* *} p<0.01$ compared with the up-regulation group ( $n=3$, student's $t$-test); $\# p<0.01$ compared with the empty and control group ( $n=3$, student's $t$-test); \#\#p<0.05 determined by one-way ANOVA and significant differences from the respective values determined by S-N-K method $(n=3)$. (C) The SOCS1 mRNA expression levels detected by real-time qPCR. ${ }^{*} p<0.01$ compared with the model up-regulation group ( $n=3$, student's $t$-test); ${ }^{* *} p<0.01$ compared with the up-regulation group $(n=3$, student's $t$-test); \#p $<0.01$ compared with the empty and control group ( $n=3$, student's $t$-test); \#\#p $<0.05$ determined by one-way ANOVA and significant differences from the respective values determined by S-N-K method $(n=3)$. (D) Effect of Corilagin on TGF- $\beta$ protein expression detected by ELISA. ${ }^{*} p<0.01$ compared with the model up-regulation group $(n=3$, student's $t$-test); ${ }^{* *} p<0.01$ compared with the up-regulation group $(n=3$, student's $t$-test); $\# p<0.01$ compared with the empty and control group $(n=3$, student's $t$-test); \#\#p<0.05 determined by one-way ANOVA and significant differences from the respective values determined by S-N-K method $(n=3)$. Data are shown with mean with SD.

\section{Statistical Analysis}

The data are presented as the means \pm SD. Referring to a previously published statistical approach (Ding et al., 2015), Kolmogorov-Smirnov (K-S) test and Shapiro-Wilk (S-W) test were performed to assess the normalcy. The significance of $\mathrm{K}-\mathrm{S}$ and $\mathrm{S}-\mathrm{W}$ test indicated that the data were normally distributed $(p>0.05)$. Levene's test was performed to assess the equality of variances. The significance of Levene's test indicated the variances of the data from different groups were equal $(p>0.05)$. The measurement data were compared between the two groups with Student's $t$-test. Multiple comparisons between multiple groups were performed with one-way ANOVA tests, 
A

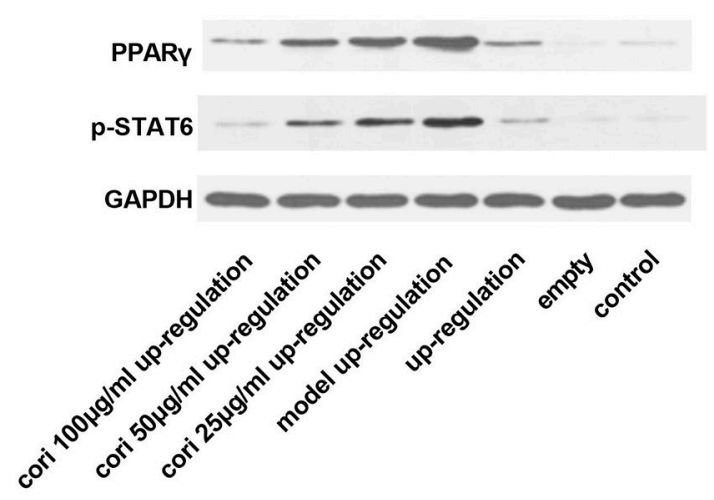

B

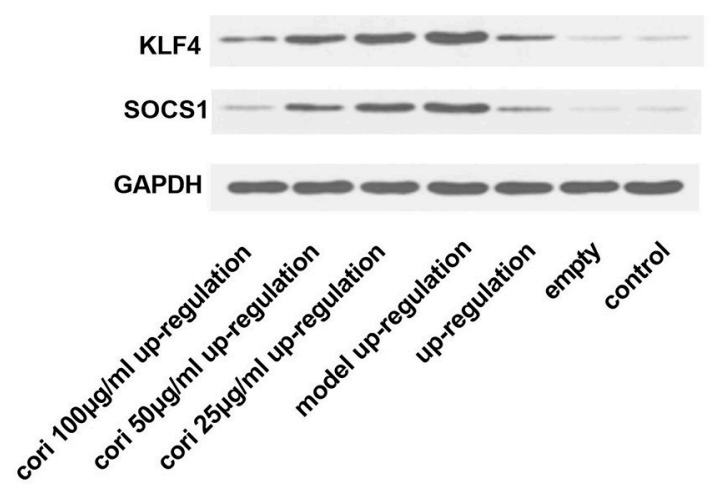

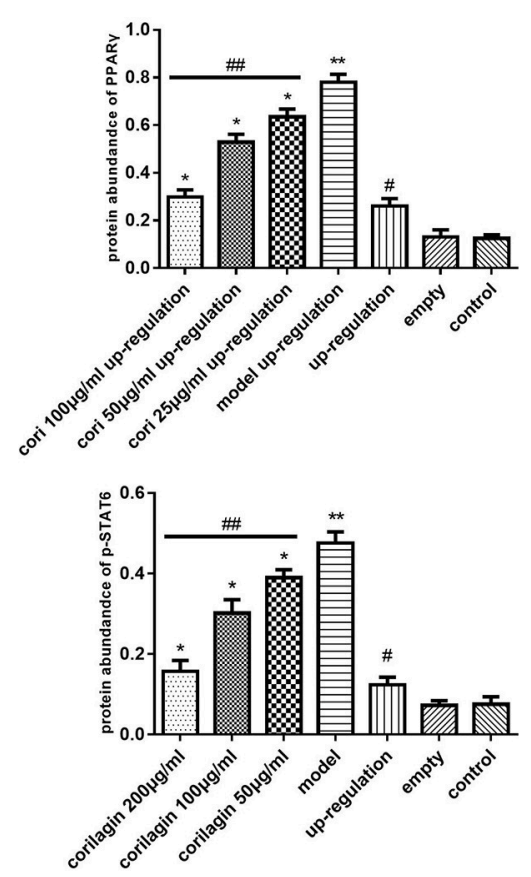
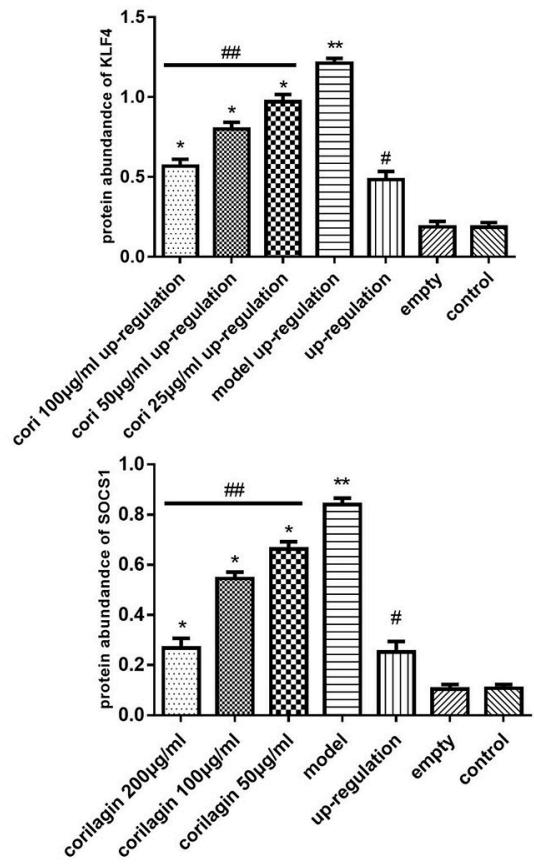

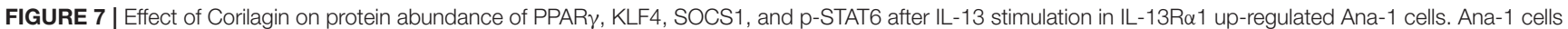
was infected with IL-13Ra1 lentivirus or empty vector for $96 \mathrm{~h}$ except control group. Subsequently, the corilagin groups and model up-regulation group were administrated with rlL-13 for $24 \mathrm{~h}$. Then the corilagin groups were treated with corilagin of different concentrations. Western blot assays were performed. The dose-dependent anti-fibrogenesis effect of corilagin still persisted when IL-13R $\alpha 1$ was up-regulated. (A) The PPAR $\gamma$ and p-STAT6 protein abundance detected by western blot assay. ${ }^{*} p<0.01$ compared with the model up-regulation group $\left(n=3\right.$, student's $t$-test); ${ }^{* *} p<0.01$ compared with the up-regulation group ( $n=3$, student's $t$-test); \#p < 0.01 compared with the empty and control group $(n=3$, student's $t$-test); \#\#p < 0.05 determined by one-way ANOVA and significant differences from the respective values determined by S-N-K method $(n=3)$. (B) The KLF4 and SOCS1 protein abundance detected by western blot assay. ${ }^{*} p<0.01$ compared with the model up-regulation group $\left(n=3\right.$, student's $t$-test); ${ }^{* *} p<0.01$ compared with the up-regulation group $(n=3$, student's $t$-test); \#p $<0.01$ compared with the empty and control group $(n=3$, student's $t$-test); \#\#p < 0.05 determined by one-way ANOVA and significant differences from the respective values determined by S-N-K method $(n=3)$. Data are shown with mean with SD. 

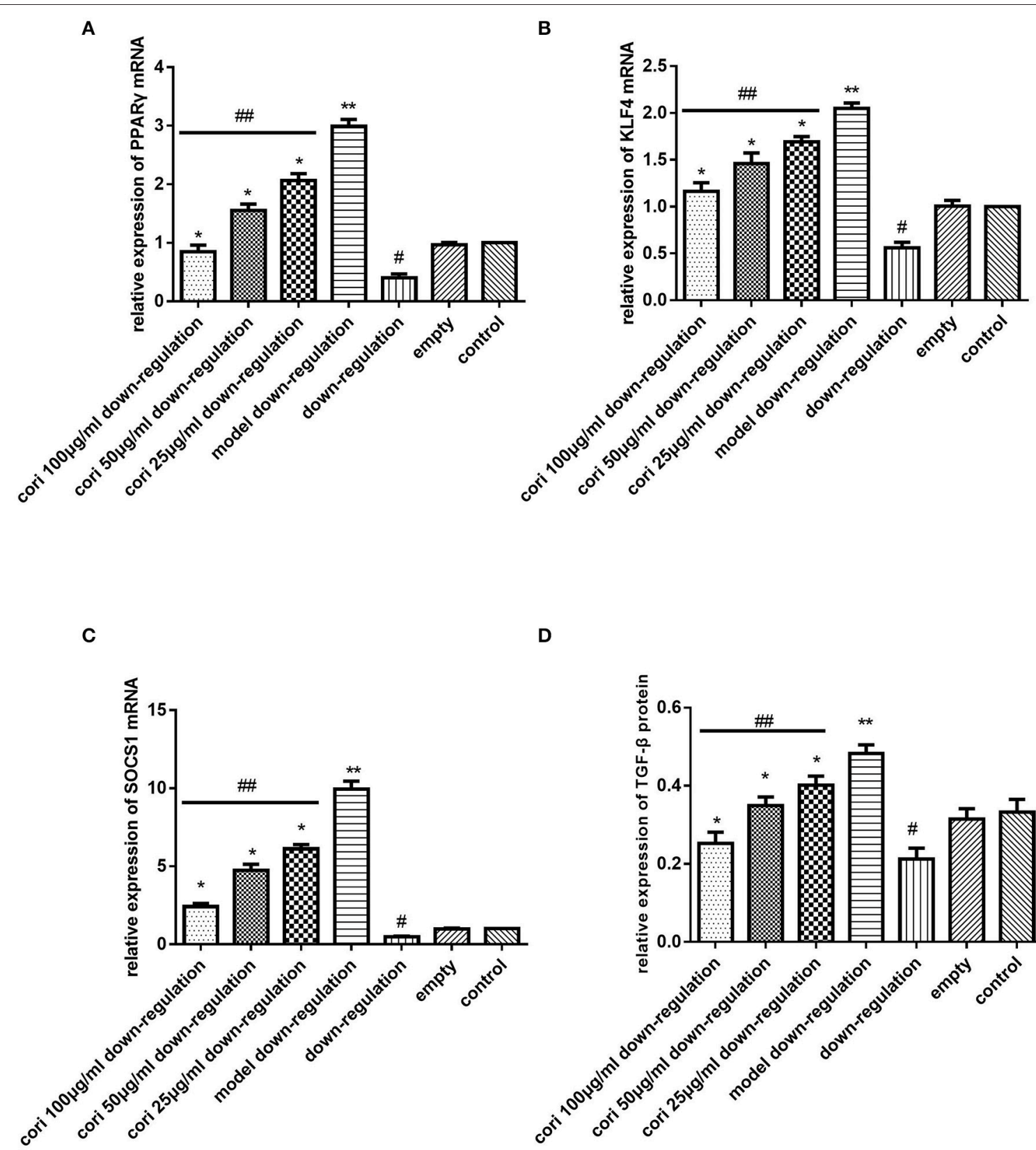

D

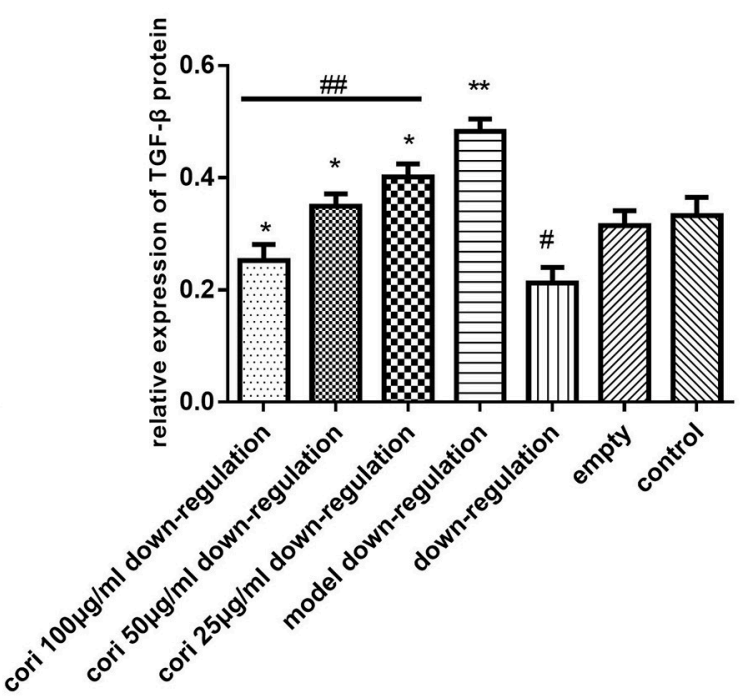

FIGURE 8 | Effect of Corilagin on mRNA expression of PPAR $\gamma$, KLF4, SOCS1, IL-13R $\alpha$ 1, STAT6, and protein expression of TGF- $\beta$ after IL-13 stimulation in IL-13R $\alpha 1$ down-regulated Ana-1 cells. Ana-1 cells was infected with IL-13R $\alpha 1$ siRNA or empty vector for $48 \mathrm{~h}$ except control group. Subsequently, the corilagin groups and model down-regulation group were administrated with rlL-13 for $24 \mathrm{~h}$. Then the corilagin groups were treated with corilagin of different concentrations. Real-time qPCR and ELISA were performed. The dose-dependent anti-fibrogenesis effect of corilagin still persisted when IL-13R $\alpha 1$ was down-regulated. (A) The PPAR $\gamma$ mRNA expression levels detected by real-time GPCR. ${ }^{*} p<0.01$ compared with the model down-regulation group $\left(n=3\right.$, student's $t$-test); ${ }^{* *} p<0.01$ compared with the down-regulation group ( $n=3$, student's $t$-test); \#p < 0.01 compared with the empty and control group $(n=3$, student's $t$-test); \#\# $<0.05$ determined by one-way ANOVA and significant differences from the respective values determined by S-N-K method $(n=3)$. (B) The KLF4 mRNA expression levels detected by real-time GPCR. ${ }^{*} p<0.01$ compared with the model down-regulation group $\left(n=3\right.$, student's $t$-test); ${ }^{*} p<0.01$ compared with the down-regulation group $(n=3$, student's $t$-test); $\# p<0.01$ compared with the empty and control group $(n=3$, student's $t$-test); \#\#p $<0.05$ determined by one-way ANOVA and significant differences from the respective values determined by S-N-K method $(n=3)$. (C) The SOCS1 mRNA expression levels detected by real-time qPCR. ${ }^{*} p<0.01$ compared with the model down-regulation group ( $n=3$, student's $t$-test); ${ }^{* *} p<0.01$ compared with the down-regulation group $(n=3$, student's $t$-test); \#p<0.01 compared with the empty and control group $(n=3$, student's $t$-test); $\# \# p<0.05$ determined by one-way ANOVA and significant differences from the respective values determined by S-N-K method $(n=3)$. (D) Effect of Corilagin on TGF- $\beta$ protein expression detected by ELISA. ${ }^{*} p<0.01$ compared with the model down-regulation group $(n=3$, student's $t$-test); ${ }^{* *} p<0.01$ compared with the down-regulation group $(n=3$, student's $t$-test); $\# p<0.01$ compared with the empty and control group $(n=3$, student's $t$-test); \#\#p < 0.05 determined by one-way ANOVA and significant differences from the respective values determined by S-N-K method $(n=3)$. Data are shown with mean with SD. 

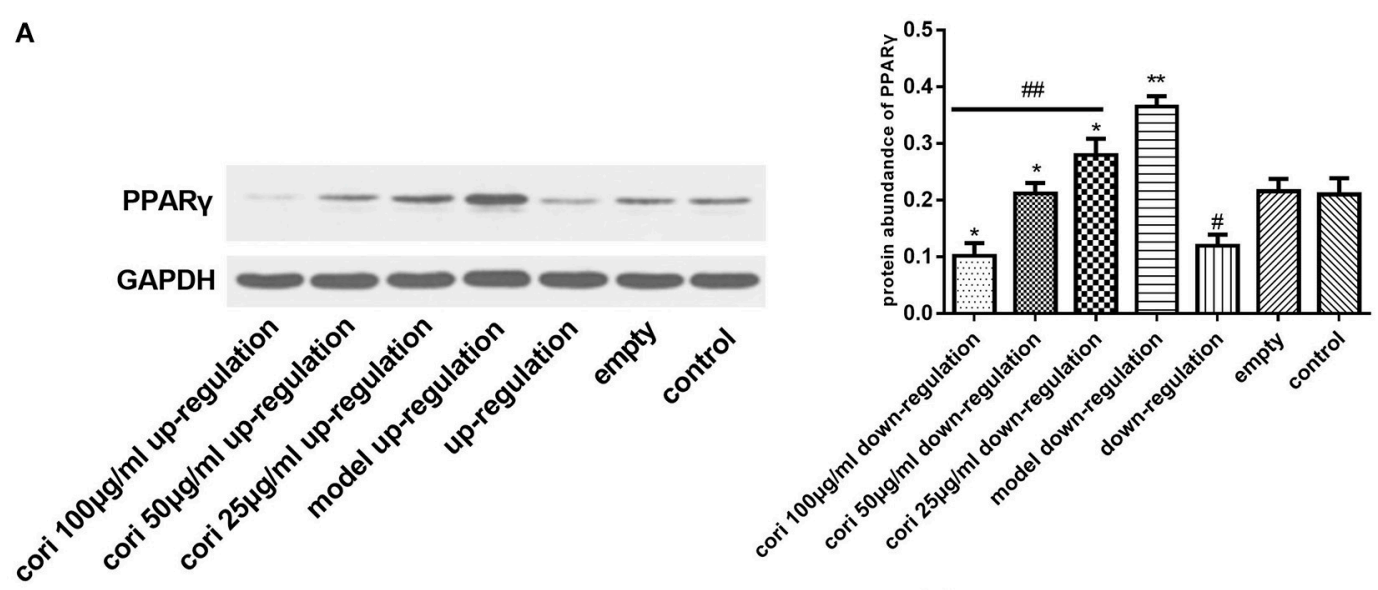

B

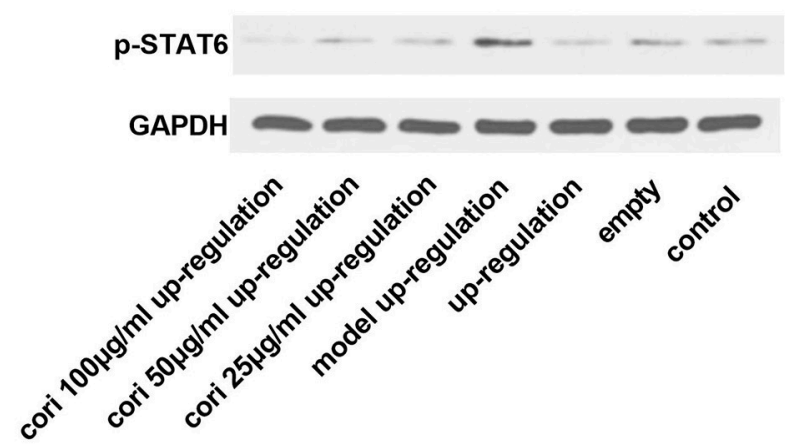

C
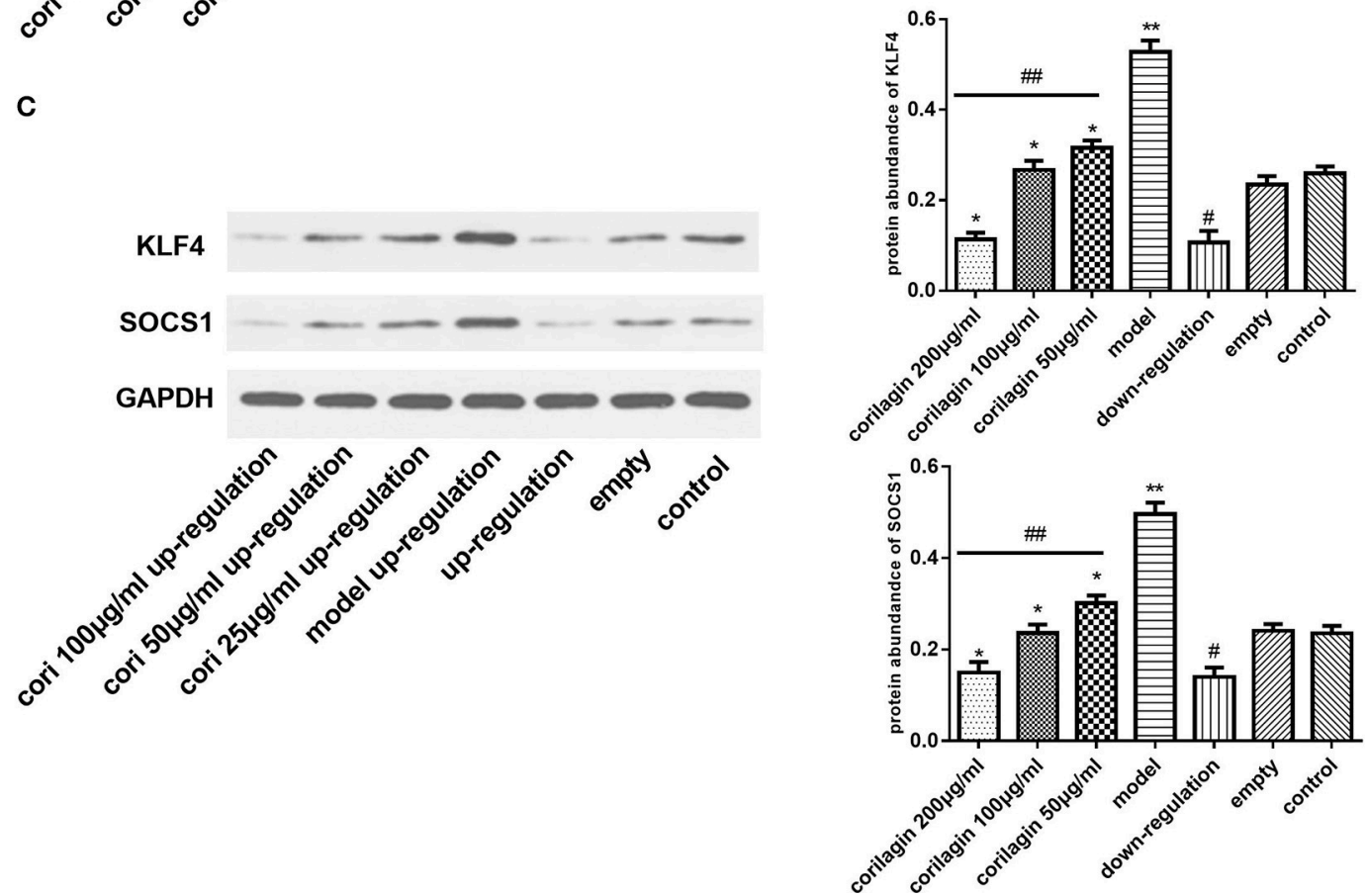

FIGURE 9 | Effect of Corilagin on protein abundance of PPAR $\gamma$, KLF4, SOCS1 and p-STAT6 after IL-13 stimulation in IL-13R 1 down-regulated Ana-1 cells. Ana-1 cells was infected with IL-13R $\alpha 1$ siRNA or empty vector for $48 \mathrm{~h}$ except control group. Subsequently, the corilagin groups and model down-regulation group were administrated with rlL-13 for $24 \mathrm{~h}$. Then the corilagin groups were treated with corilagin of different concentrations. Western blot assays were performed. The dose-dependent anti-fibrogenesis effect of corilagin still persisted when IL-13R $\alpha 1$ was down-regulated. (A) The PPAR $\gamma$ protein abundance detected by western blot 


\section{FIGURE 9 | Continued}

assay. ${ }^{*} p<0.01$ compared with the model down-regulation group $\left(n=3\right.$, student's $t$-test); ${ }^{* *} p<0.01$ compared with the down-regulation group ( $n=3$, student's $t$-test); $\# p<0.01$ compared with the empty and control group ( $n=3$, student's $t$-test); \#\#p < 0.05 determined by one-way ANOVA and significant differences from the respective values determined by S-N-K method $(n=3)$. (B) The p-STAT6 protein abundance detected by western blot assay. ${ }^{*} p<0.01$ compared with the model down-regulation group ( $n=3$, student's $t$-test); ${ }^{* *} p<0.01$ compared with the down-regulation group $(n=3$, student's $t$-test); \#p $<0.01$ compared with the empty and control group ( $n=3$, student's t-test); \#\#p < 0.05 determined by one-way ANOVA and significant differences from the respective values determined by S-N-K method $(n=3)$. (C) The KLF4 and SOCS1 protein abundance detected by western blot assay. ${ }^{*} p<0.01$ compared with the model down-regulation group $(n=3$, student's $t$-test); ${ }^{\star \star} p<0.01$ compared with the down-regulation group $(n=3$, student's $t$-test); $\# p<0.01$ compared with the empty and control group $(n=3$, student's $t$-test); \#\#p < 0.05 determined by one-way ANOVA and significant differences from the respective values determined by S-N-K method $(n=3)$. Data are shown with mean with SD.

and a post-hoc test was performed by the Student-NewmanKeuls (S-N-K) method. The statistical analyses were conducted with SPSS 12.0 software. Statistical significance was defined at $p<0.05$.

\section{RESULTS}

\section{Cytotoxicity of Corilagin in Ana-1 Cells}

The viability of Ana-1 cells with corilagin administration was illustrated in Figure 1. As illustrated, the viabilities of all corilagin groups decreased compared with the $0 \mu \mathrm{g} / \mathrm{ml}$ corilagin group ( $p<0.05$, Student's $t$-test, $n=3$ ). In our study, we chose the concentrations which could result in a cell viability more than $70 \%(100,50,25, \mu \mathrm{g} / \mathrm{ml})$. With the corilagin concentration ranging from 50 to $400 \mu \mathrm{g} / \mathrm{ml}$, the viability decreased with increasing concentrations of corilagin $(p<0.05$, one-way ANOVA, post-hoc: S-N-K method, $n=3)$, suggesting a dosedependent effect.

\section{Effect of Corilagin on IL-13R $\alpha 1$ Signaling Pathway after IL-13 Stimulation in Ana-1 Cells}

PPAR $\gamma$, KLF4, SOCS1, and p-STAT6 are important regulators of M2 macrophage polarization in the IL- $13 \alpha 1$ signaling pathway (Sica and Mantovani, 2012). Therefore, we investigated the expression of genes besides IL-13R $\alpha 1$ to explore the antifibrogenic effect of corilagin. As illustrated in Figure 2, the mRNA levels of PPAR $\gamma, \mathrm{KLF} 4$ and SOCS1 were detected by realtime qPCR (Figures $\mathbf{2 A - C , G}$ ), and the TGF- $\beta$ levels in the cell supernatant were detected by ELISA (Figure 2F). Meanwhile, we detected mRNA levels of IL-13R $\alpha 1$ and STAT6 by realtime qPCR (Figures 2D,E). The abundance of PPAR $\gamma, \mathrm{KLF} 4$, SOCS1, and p-STAT6 proteins was detected by Western blot (Figure 3). The levels of PPAR $\gamma, \mathrm{KLF} 4$, SOCS1, and p-STAT6 in the model experimental groups were increased compared with levels in the control group ( $p<0.01$, Student's $t$-test, $n=3$ ), which revealed the model is successful and the expression of these molecules increased with IL-13 stimulation. Meanwhile, with corilagin treatment after IL-13 stimulation, the levels of these molecules decreased significantly compared with the levels in the model group ( $p<0.01$ or 0.05 , Student's $t$-test, $n=$ $3)$. And the levels decreased with increasing concentrations of corilagin ( $p<0.05$, one-way ANOVA, post-hoc: S-N-K method, $n=3$ ), which confirmed a dose-dependent inhibitory effect of corilagin on the cytokines in IL-13R $\alpha 1$ signaling pathway in vitro. With praziquantel treatment after IL-13 stimulation, the levels of these molecules showed no significant difference compared with the model group ( $p>0.05$, Student's $t$-test, $n=3$ ), which indicated praziquantel had little effect on the cytokines in IL$13 \mathrm{R} \alpha 1$ signaling pathway. There were no significant differences in the levels of IL-13R $\alpha 1$ and STAT6 among groups $(p>$ 0.05). As illustrated in Figures 2B,G, the trends of KLF4 mRNA expression were showed to be consistent when real-time qPCR was performed with two reference genes in our study.

\section{Effect of Up-Regulation of IL-13R $\alpha 1$ via Lentivirus Transfection}

To further explore the anti-fibrotic effect of corilagin in hepatic schistosomiasis, we up-regulated the expression of IL-13R $\alpha 1$ by lentivirus transfection. The transfection effect was observed under a fluorescence microscope and examined by real-time qPCR, ELISA and Western blot. The Ana-1 cells were observed under a fluorescence microscope $96 \mathrm{~h}$ after lentivirus transfection (Figure 4A). The mRNA levels of IL-13 $\alpha 1$ were determined by real-time $\mathrm{qPCR}$, and the expression of IL-13R $\alpha 1$ in upregulation group increased by $130 \%$ compared to the control group (Figure 4B). The abundance of IL-13 $\alpha 1$ protein was determined by Western blot, and the abundance of IL-13R $\alpha 1$ protein in up-regulation group increased by $115 \%$ compared to the control group (Figure 4C). As illustrated, the fluorescence was strong and most cells were infected successfully. The levels of IL-13R $\alpha 1$ mRNA and protein in the up-regulation group were increased significantly compared with the control groups $(p<0.01$, Student's $t$-test, $n=3)$. The levels in the empty-vector group showed no significant difference compared to the control group ( $p>0.05$, Student's $t$-test, $n=3$ ).

\section{Effect of Down-Regulation of IL-13R $\alpha 1$ via siRNA Transfection}

We down-regulated the expression of IL-13R $\alpha 1$ by siRNA transfection. The transfection effect was observed under a fluorescence microscope and examined by real-time qPCR, ELISA and Western blot. The Ana-1 cells were observed under a fluorescence microscope $48 \mathrm{~h}$ after the siRNA transfection (Figure 5A). As illustrated, the fluorescence was strong and most cells were infected successfully. The mRNA levels of IL-13 $\alpha 1$ were determined by real-time qPCR, and the expression of IL$13 \mathrm{R} \alpha 1$ in down-regulation group dropped $61 \%$ compared to 

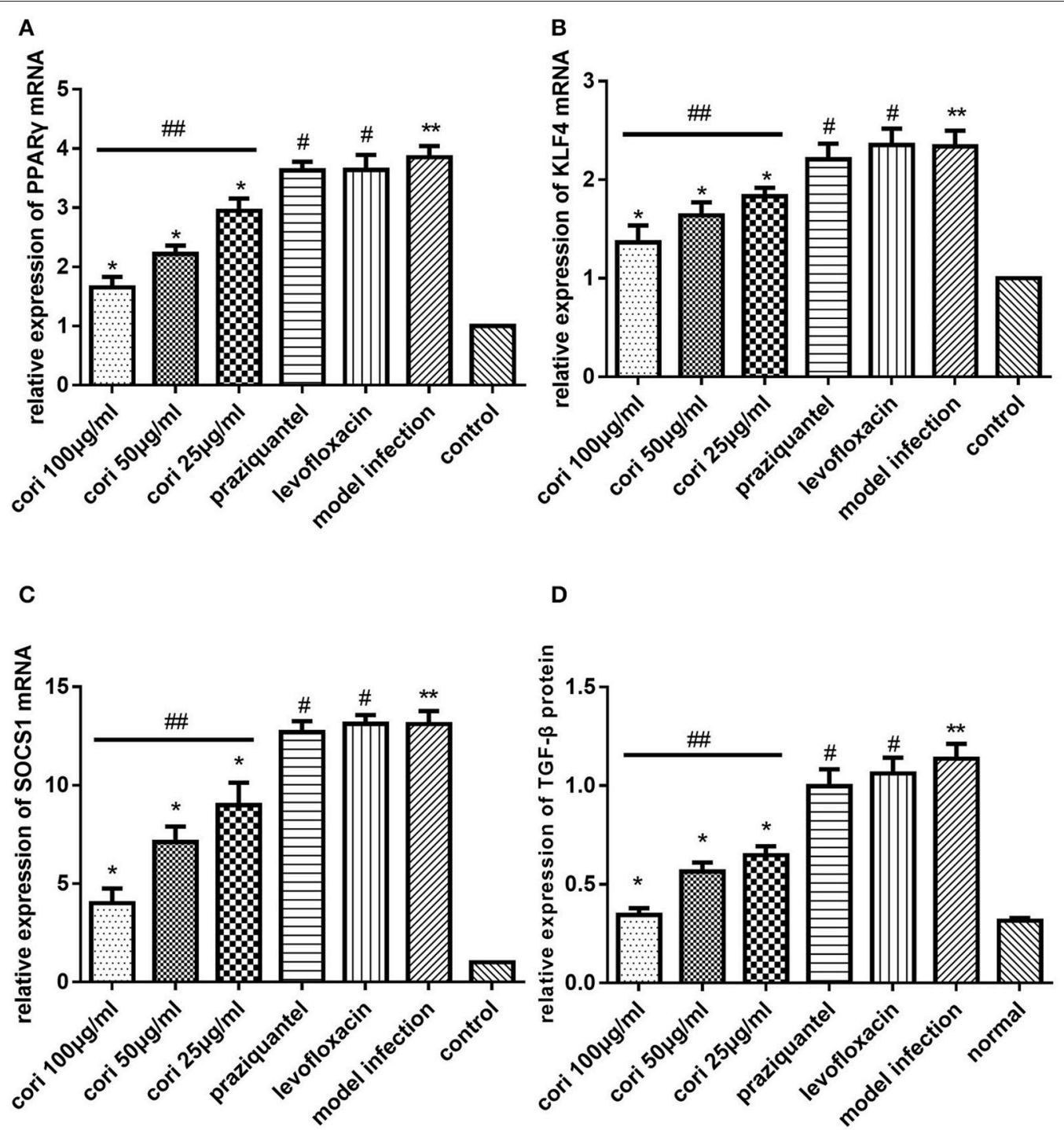

FIGURE 10 | Effect of Corilagin on mRNA expression of PPAR $\gamma$, KLF4, SOCS1, and protein expression of TGF- $\beta$ in animal model. Twenty-eight days after the mice were infected with cercaria, 5-day praziquantel treatment was administrated to kill the adult schistosomes. Then the corilagin groups, praziquantel group and levofloxacin group were administrated with different drugs respectively for 21 days. Real-time GPCR and ELISA were performed. Corilagin showed a significant dose-dependent inhibitory effect on IL-13R $\alpha 1$ signaling pathway in vivo. However, the praziquantel treatment had little effect. (A) The PPAR $\gamma$ mRNA expression levels detected by real-time qPCR. ${ }^{*} p<0.01$ compared with the model infection group $\left(n=6\right.$, student's $t$-test); ${ }^{* *} p<0.01$ compared with the control group $(n=6$, student's $t$-test); $\# p<0.01$ compared with the control group ( $n=6$, student's $t$-test); \#\#p < 0.05 determined by one-way ANOVA and significant differences from the respective values determined by S-N-K method $(n=6)$. (B) The KLF4 mRNA expression levels detected by real-time qPCR. ${ }^{*} p<0.01$ compared with the model infection group ( $n=6$, student's $t$-test); ${ }^{* *} p<0.01$ compared with the control group $(n=6$, student's $t$-test); $\# p<0.01$ compared with the control group $(n=6$, student's $t$-test); \#\#p < 0.05 determined by one-way ANOVA and significant differences from the respective values determined by S-N-K method ( $n=6$ ). (C) The SOCS1 mRNA expression levels detected by real-time GPCR. ${ }^{*} p<0.01$ compared with the model infection group $\left(n=6\right.$, student's $t$-test); ${ }^{* \star} p<0.01$ compared with the control group ( $n=6$, student's $t$-test); $\# p<0.01$ compared with the control group ( $n=6$, student's $t$-test); \#\#p $<0.05$ determined by one-way ANOVA and significant differences from the respective values determined by S-N-K method $(n=6)$. (D) Effect of Corilagin on TGF- $\beta$ protein expression detected by ELISA. ${ }^{*} p<0.01$ compared with the model infection group $\left(n=6\right.$, student's $t$-test); ${ }^{* *} p<0.01$ compared with the control group $(n=6$, student's $t$-test); $\# p<0.01$ compared with the control group ( $n=6$, student's $t$-test); \#\#p $<0.05$ determined by one-way ANOVA and significant differences from the respective values determined by S-N-K method $(n=6)$. Data are shown with mean with SD.

the control group (Figure 5B). The abundance of IL-13 $\alpha 1$ was determined by Western blot, and the abundance of IL-13R $\alpha 1$ in down-regulation group dropped $41 \%$ (Figure 5C). As shown in Figure 5, the level of IL-13R $\alpha 1$ in the down-regulation group was decreased significantly compared with the empty-vector and control groups ( $p<0.01$, Student's $t$-test, $n=3)$. The level in the empty-vector group showed no significant differences compared with that in the control group ( $p>0.05$, Student's $t$-test, $n=3$ ). 

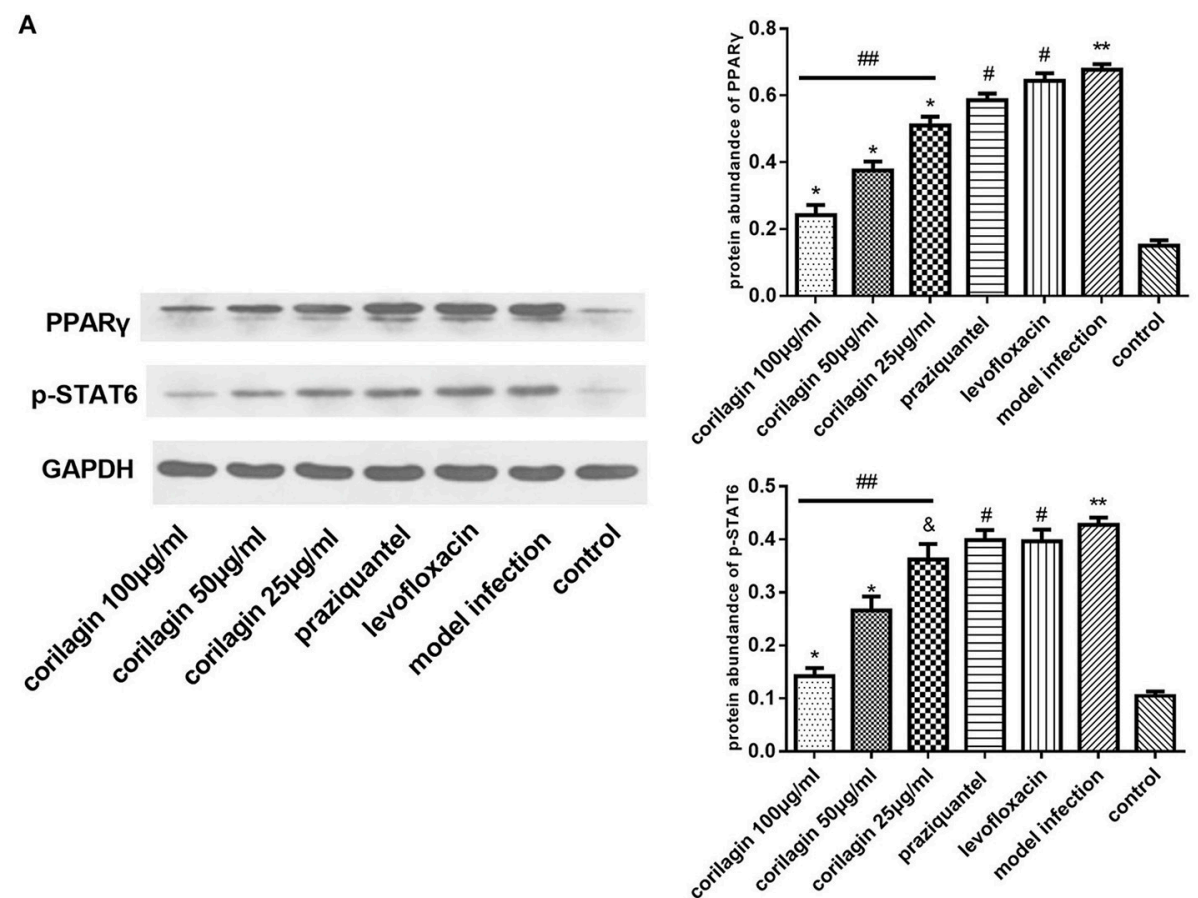

B
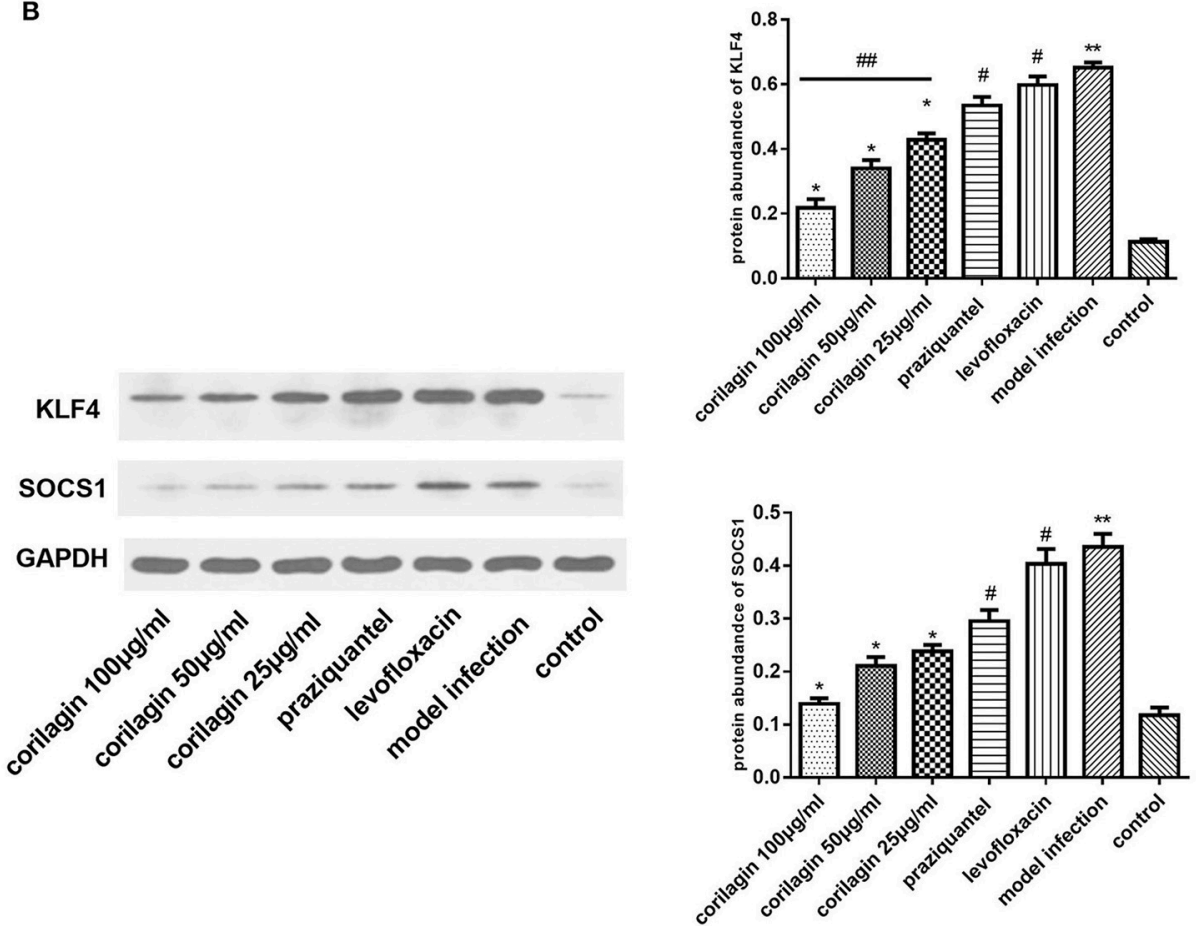

FIGURE 11 | Effect of Corilagin on protein abundance of PPAR $\gamma$, KLF4, SOCS1, and p-STAT6 in animal model. After the mice model was established as we stated, western blot assays were performed. Corilagin showed a significant dose-dependent inhibitory effect on IL-13Ra 1 signaling pathway in vivo. However, the praziquantel treatment had little effect. (A) The PPAR $\gamma$ and p-STAT6 protein abundance detected by western blot assay. ${ }^{*} p<0.01$ compared with the model infection group ( $n=6$, student's $t$-test); \& $p<0.05$ compared with the model infection group $\left(n=6\right.$, student's $t$-test); ${ }^{*} p<0.01$ compared with the control group $(n=6$, student's $t$-test); $\# p<0.01$ compared with the control group $(n=6$, student's $t$-test); \#\#p < 0.05 determined by one-way ANOVA and significant differences from the respective values determined by S-N-K method $(n=6)$. (B) The KLF4 and SOCS1 protein abundance detected by western blot assay. ${ }^{*} p<0.01$ compared with the model infection group ( $n=6$, student's $t$-test); ${ }^{*} p<0.01$ compared with the control group $(n=6$, student's $t$-test); \#p $<0.01$ compared with the control group $(n=$ 6 , student's t-test); \#\#p < 0.05 determined by one-way ANOVA and significant differences from the respective values determined by S-N-K method $(n=6)$. Data are shown with mean with SD. 

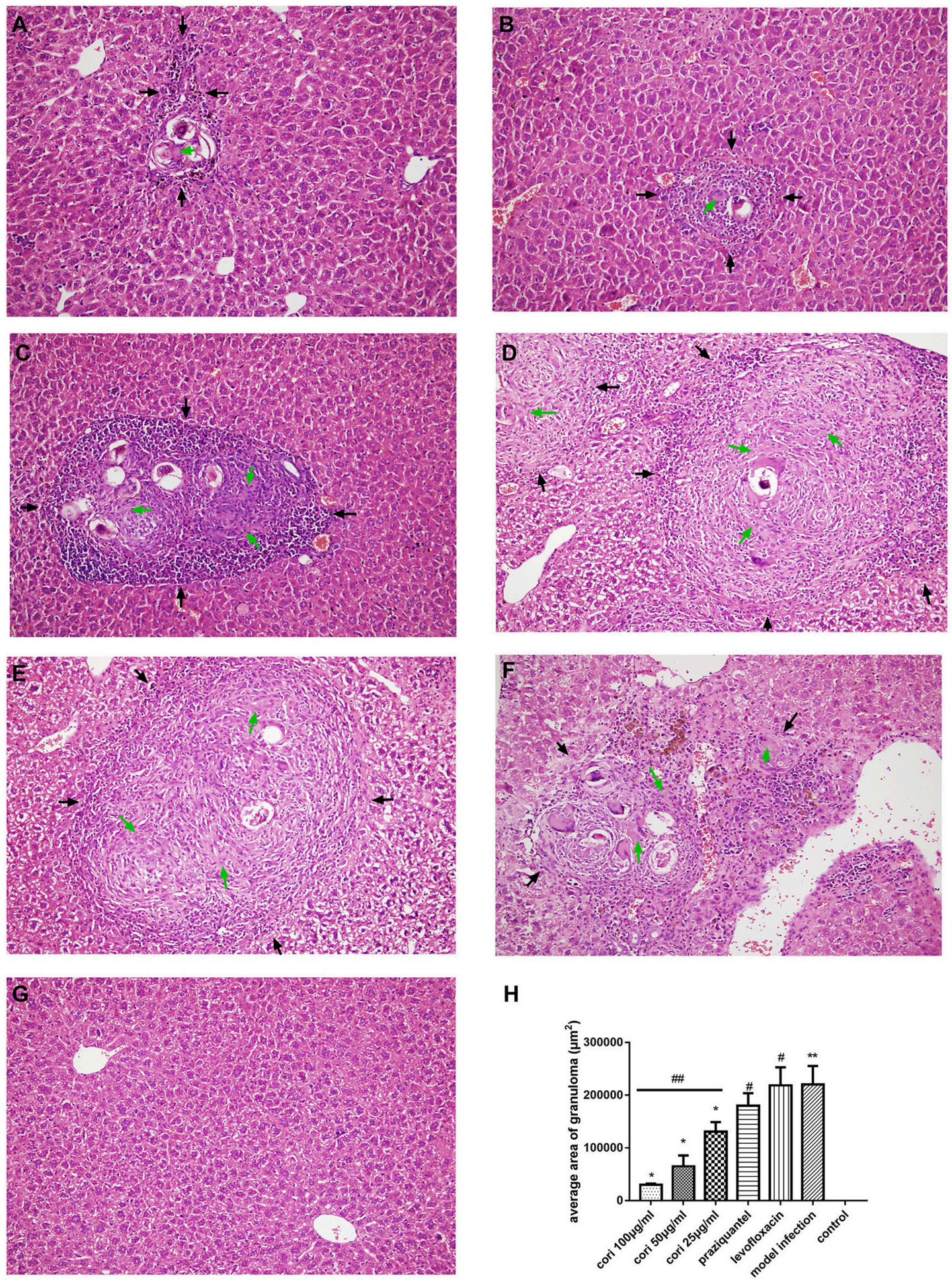

FIGURE 12 | Effect of Corilagin on schistosomiasis fibrosis in animal model examined by HE staining in 200× magnification. After the mice model was established, $\mathrm{HE}$ staining was performed. In each figure, the black arrows indicate the area of fibrotic nodules and the green arrows indicate the fibrin which was stained pink in HE staining. Corilagin treatment significantly reduced the granuloma area in vivo. However, the praziquantel treatment had little effect. (A) High-concentration corilagin group; (B) medium-concentration corilagin group; (C) low-concentration corilagin group; (D) praziquantel group; (E) levofloxacin group; (F) model infection group; (G) control group; $\mathbf{( H )}$ average area of granuloma. ${ }^{*} p<0.01$ compared with the model infection group $\left(n=6\right.$, student's $t$-test); ${ }^{* *} p<0.05$ compared with the control group ( $n=6$, student's $t$-test); \#p < 0.01 compared with the control group ( $n=6$, student's $t$-test); \#\# $<0.05$ determined by one-way ANOVA and significant differences from the respective values determined by S-N-K method $(n=6)$. Data are shown with mean with SD. 

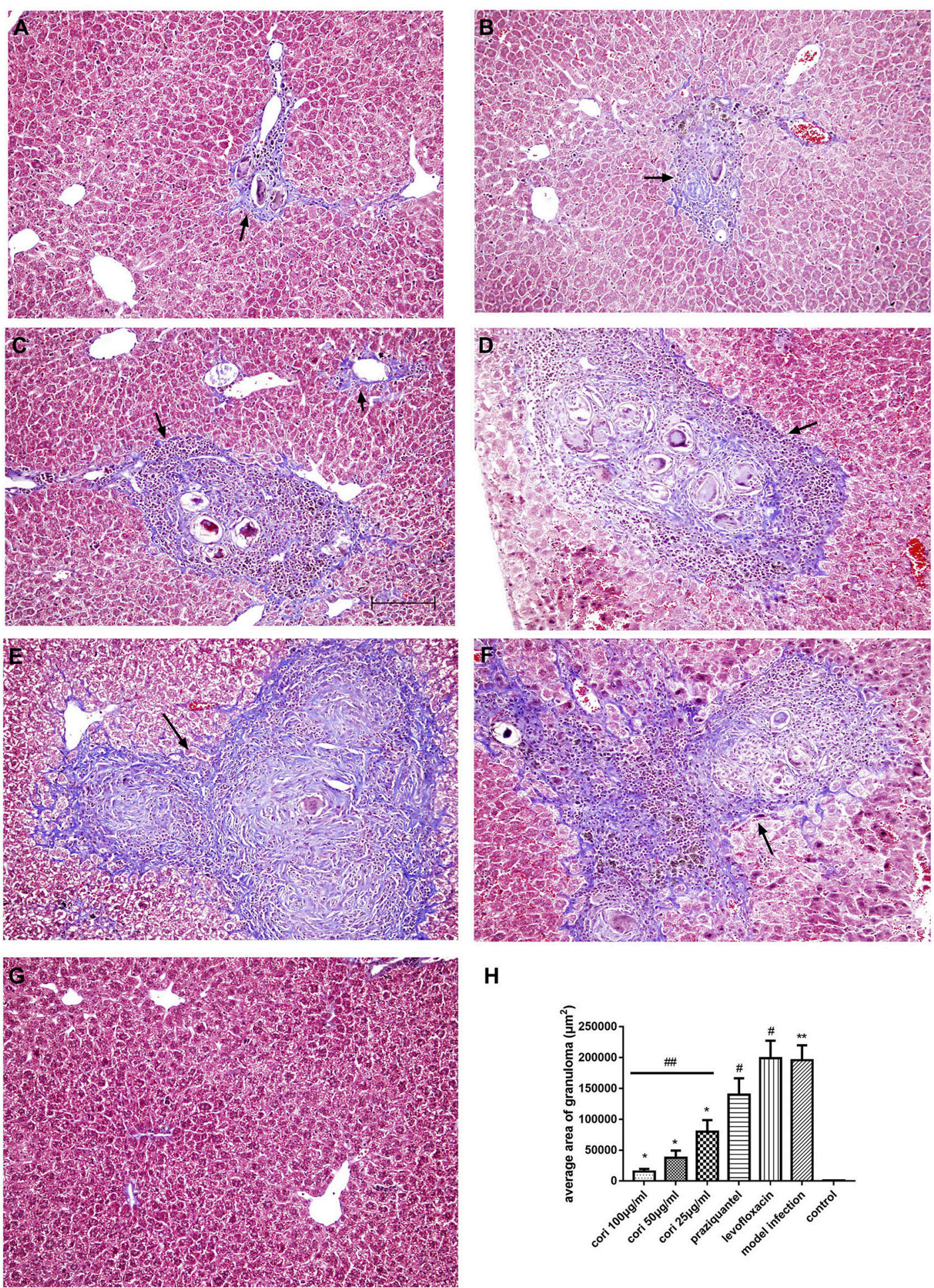

H

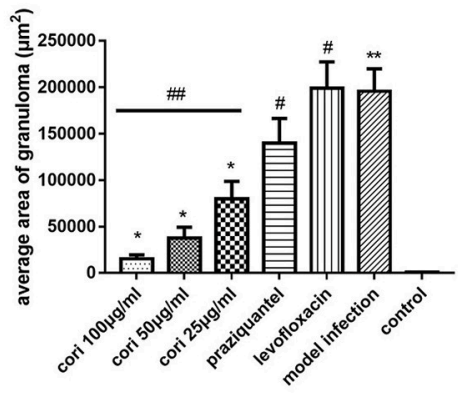

FIGURE 13 | Effect of Corilagin on schistosomiasis fibrosis in animal model examined by Masson staining in 200x magnification. After the mice model was established, HE staining was performed. Black arrows indicate the area of fibrosis which was stained purple in Masson staining. Corilagin treatment significantly reduced the fibrosis area in vivo. However, the praziquantel treatment had little effect. (A) high-concentration corilagin group; (B) medium-concentration corilagin group; (C) low-concentration corilagin group; (D) praziquantel group; (E) levofloxacin group; (F) model infection group; (G) control group; (H) average area of fibrosis. ${ }^{*} p<0.05$ compared with the model infection group $\left(n=6\right.$, student's $t$-test); ${ }^{* *} p<0.01$ compared with the control group $(n=6$, student's $t$-test); \#p $<0.01$ compared with the control group $(n=6$, student's $t$-test); \#\# $<0.05$ determined by one-way ANOVA and significant differences from the respective values determined by S-N-K method $(n=6)$. Data are shown with mean with SD. 


\section{Effect of Corilagin on the IL-13R $\alpha 1$ Signaling Pathway after IL-13 Stimulation in IL-13R $\alpha 1$ Up-Regulated and Down-Regulated Ana-1 Cells}

Real-time qPCR was performed to examine the mRNA expression levels of PPAR $\gamma, \mathrm{KLF} 4$ and SOCS1 in IL-13R $\alpha 1$ upregulated Ana-1 cells (Figures 6A-C) and down-regulated Ana-1 cells (Figures 8A-C). The TGF- $\beta$ levels in the cell supernatant were determined by ELISA (Figures 6D, 8D). The abundance of PPAR $\gamma$, KLF4, SOCS1 and p-STAT6 was determined by Western blot (Figures 7, 9). As illustrated, the levels of these molecules in the up- or down-regulation group correspondingly increased or decreased significantly compared with the control group $(p<0.01)$, indicating that IL-13R $\alpha 1$ up- or down-regulation had a significant effect on the IL- $13 \alpha 1$ signaling pathway. With IL13 stimulation, the levels in the model up- or down-regulation group were increased significantly compared with those in the up- or down-regulation group ( $p<0.01$, Student's $t$-test, $n=3$ ). The levels of these molecules with corilagin treatment of different concentrations were decreased compared with the model up- or down-regulation group ( $p<0.01$, Student's $t$-test, $n=3$ ), and the inhibitory effects increased with the increasing concentration of corilagin ( $p<0.05$, one-way ANOVA, post-hoc: S-N-K method, $n=3$ ), which revealed the dose-dependent inhibitory effect of corilagin on IL-13R $\alpha 1$ signaling pathway still persisted when IL$13 \mathrm{R} \alpha 1$ was up- or down-regulated. The levels of these molecules in the empty-vector group showed no significant differences compared with the control group $(p>0.05$, Student's $t$-test, $n=3)$.

\section{Effect of Corilagin on IL-13R $\alpha 1$ Signaling Pathway after Praziquantel Administration in Schistosomiasis C57BL/6 Mice}

In the animal model, we quantified the mRNA expression of PPAR $\gamma, \mathrm{KLF} 4$ and SOCS1 in liver tissue using real-time qPCR (Figures 10A-C). The level of TGF- $\beta$ in mice serum was measured by ELISA (Figure 10D). The abundance of PPAR $\gamma$, KLF4, SOCS1 and p-STAT6 in liver tissue was measured by Western blot (Figure 11). As shown, the levels of these molecules in the model infection group were increased compared with the control group ( $p<0.01$, Student's $t$-test, $n=6$ ). The levels of these molecules with corilagin treatment of different concentrations were decreased compared with the model infection group ( $p<0.01$ or $p<0.05$, Student's $t$-test, $n=6$ ), and the inhibitory effects increased with increasing concentrations of corilagin ( $p<0.05$, one-way ANOVA, posthoc: S-N-K method, $n=6$ ), which confirmed the dose-dependent inhibitory effect of corilagin on IL-13R $\alpha 1$ signaling pathway in vivo. The levels of these molecules in the praziquantel group (positive control) and levofloxacin group (negative control) were increased compared with the control group $(p<0.01$, Student's $t$-test, $n=6)$ and showed no significant differences compared with the model infection group $(p>0.05$, Student's $t$-test, $n=6$ ), which indicated praziquantel had little effect on IL-13R $\alpha 1$ signaling pathway.

\section{Effect of Corilagin on Pathological Changes of Schistosomiasis Liver Fibrosis in the Mouse Liver}

The effect of corilagin on pathological changes of schistosomiasis liver fibrosis in mice liver was observed with HE, Masson staining and immunohistochemistry. According to the results, all the liver tissue of the infected mice showed varying degrees of fibrosiss, which indicated the model establishment was successful. As illustrated in Figure 12, significant fibrosis and granuloma were observed in the model infection, praziquantel and levofloxacin groups, and the area of granuloma was remarkably larger than that in the control group ( $p<0.01$, Student's $t$-test, $n=6$ ). The area of granuloma with corilagin treatment of different concentrations compared was significantly smaller with the model infection groups ( $p<0.05$, Student's $t$-test, $n=6$ ), and the area decreased with increasing concentrations of corilagin $(p<0.05$, one-way ANOVA, post-hoc: S-N-K method, $n=6)$, which showed a dose-dependent anti-fibrogenesis effect. For pathological scores, apart from normal group, the inflammation score of high-concentration corilagin group and mediumconcentration corilagin group was G2, while low-concentration corilagin group was G2-G3, and praziquantel group was G3. The inflammation score of levofloxacin group and model infection group was G3-G4. The fibrosis score of corilagin groups was S2 and praziquantel, levofloxacin group and model infection group was S3. As illustrated in Figure 13, and the area of fibrosis in the model infection, praziquantel and levofloxacin groups was remarkably larger than that in the control group $(p<0.01$, Student's $t$-test, $n=6$ ). The area of fibrosis with corilagin treatment of different concentrations compared was significantly smaller compared with the model infection groups $(p<0.05$, Student's $t$-test, $n=6$ ), and the area decreased with increasing concentrations of corilagin ( $p<0.05$, one-way ANOVA, post-hoc: S-N-K method, $n=6$ ), which showed a dose-dependent antifibrogenesis effect. As illustrated in Figure 14, the levels of CD206 staining in the model infection, praziquantel and levofloxacin groups were significantly higher than that in the control group $(p<0.01$, Student's $t$-test, $n=6)$. The levels of CD206 staining were significantly lower in the corilagin groups compared with the model infection groups ( $p<0.05$, Student's $t$-test, $n=6$ ), and the area decreased with increasing concentrations of corilagin $(p<0.05$, one-way ANOVA, post-hoc: S-N-K method, $n=6)$. The less area of fibrosis and distribution of M2 macrophages in corilagin treatment group indicated the anti-fibrogenic effect of corlagin in vivo.

\section{DISCUSSION}

Schistosomiasis hepatic fibrosis is a complex pathological process involving multiple cell-signaling pathways and cytokines. M2 macrophages play a critical role in the pathogenesis of this disease ( $\mathrm{He}$ et al., 2016). M2 macrophages are produced upon the stimulation of IL-13 (Gordon, 2003), which is significantly up-regulated during schistosome infection. The expression of M2 macrophage markers such as Arg-1, Ym-1, and Fizz-1 were highly increased in schistosomiasis (Sandler 
et al., 2003). These markers induce L-arginine to be converted to proline, an essential amino acid for the production of collagen (Hesse et al., 2001). Apart from the pro-fibrotic cytokines produced by M2 macrophages, M2 macrophages are an important component of granuloma in schistosomiasis (Ragheb and Boros, 1989).

M2 polarization of macrophages is induced by the IL13-mediated Th2 immune response and eventually leads to the secretion of pro-fibrotic cytokines. IL-13 binding to the IL-4R $\alpha /$ IL-13R $\alpha 1$ receptor complex triggers phosphorylation of STAT6, further promoting the expression of downstream profibrotic molecules (Anthony et al., 2012). In this study, we aimed to explore the effects of corilagin in controlling egg-induced hepatic fibrosis after the adult schistosomes were killed and to detect the expression of IL-13 $\alpha 1$ and the downstream M2 molecules, which suggested that the mechanism of suppressing egg-induced hepatic fibrosis was mediated by inhibiting the activation of IL-13 $\alpha 1$ signaling pathway and M2 polarization of macrophages.

IL-13R $\alpha 1$ is a central mediator of the Th2-biased response and schistosomiasis hepatic fibrosis (Beschin et al., 2013). Thus, we up- and down-regulated the expression of IL-13R $\alpha 1$ in Ana-1 cells, attempting to evaluate the effect of corilagin in the case that the sensitivity of Ana- 1 cells to IL-13 was changed. We found that the levels of IL-13R $\alpha 1$ mRNAs showed no significant differences in the model group compared with the corilagin groups and the control group, which indicated corilagin had no significant effect on the amount of IL-13R $\alpha 1$. Meanwhile, the expression levels of other signaling molecules in IL-13R $\alpha 1$ pathway such as PPAR $\gamma$, KLF4, SOCS1, p-STAT6 and TGF- $\beta$ were significantly suppressed in the corilagin groups compared with the model group. It is known that IL-13R $\alpha 1$ has a much lower affinity for IL-13 than IL-13R $\alpha 2$, but the affinity is greatly enhanced when it dimerizes with IL- $4 \mathrm{R} \alpha$ to form a receptor complex (Wang et al., 2011). Therefore, we speculate that the inhibitory effect of corilagin on the fibrosis induced by IL-13 is not achieved through altering the expression of IL-13R $\alpha 1$. Instead, corilagin might reduce the affinity of IL-13R $\alpha 1$ for IL-13 or interfere with the binding of IL-13 to IL-13R $\alpha 1$. However, these assumptions remain to be confirmed by further experiments. In IL-13R $\alpha 1$ up-/down-regulation experiments, it revealed that no matter how the levels of IL-13R $\alpha 1$ changed, corilagin still showed a significant inhibitory effect on pro-fibrotic cytokines.

Besides IL-13R $\alpha 1$, we measured the expression of other important molecules in IL-13R $\alpha 1$ signaling pathway. As one of the key transcription factors in the IL-13 $\alpha 1$ signaling pathway, STAT6 plays an important role in fibrosis in multiple organs (Edwards, 2015; Su et al., 2015). PPAR $\gamma$, KLF4 and TGF- $\beta$ are also the important pro-fibrotic downstream molecules in the IL-13 $\alpha 1$ pathway. PPAR $\gamma$ is involved in IL-13 mediated fibrogenesis in multiple organs (Bou Saab et al., 2016; $\mathrm{Yu}$ et al., 2016) and in M2 polarization of macrophages (Assuncao et al., 2017). Several studies have demonstrated that inhibition of PPAR $\gamma$ can alleviate schistosomiasis hepatic fibrosis (Attia et al., 2013; Duan et al., 2014). KLF4 is a critical molecule in M2 macrophage polarization and can inhibit M1 macrophage polarization; both processes promote fibrosis (Sica and Mantovani, 2012; Ke et al., 2015). TGF- $\beta$ can be induced by SEA and strongly stimulates the secretion of collagen (Baghy et al., 2012; Dooley and ten Dijke, 2012). We found that STAT6 phosphorylation, PPAR $\gamma$, KLF4 and TGF- $\beta$ were reduced by corilagin treatment. In cellular and animal experiments, the praziquantel group showed no significant differences compared to the model group, which indicated that praziquantel did not inhibit the IL-13 signaling pathway. Decreased expression of these pro-fibrotic molecules with corilagin administration suggests an inhibitory effect of corilagin on IL-13-induced M2 polarization of macrophages and fibrogenesis.

SOCS1 is an important negative-feedback regulator of STAT6 phosphorylation that competes with STAT6 for phosphorylation binding sites of JAK (Su et al., 2015; Kandhi et al., 2016). PPAR $\gamma$ and SOCS1 expression by macrophages increased with IL-4 stimulation (Su et al., 2015). The expression of SOCS1 increased notably following administration of IL-13, probably as a consequence of feedback from increased STAT6 phosphorylation. SOCS1 expression increases with increasing phosphorylation of STAT6, as a part of negative-feedback regulation of p-STAT6.

To confirm the inhibitory effect of corilagin on egg-induced fibrosis in vivo, we observed pathological changes in the mouse liver by examining tissue sections in animal experiments. HE staining was performed to observe the morphological characteristics of the cells and the area of granuloma, and Masson staining was performed to observe the degree of fibrosis in the area of deep staining. It is admitted that CD206 is a hallmark of M2 macrophage (Wollenberg et al., 2002; Aron-Wisnewsky et al., 2009; Biswas and Mantovani, 2010). Therefore, immunohistochemistry for CD206 was performed to observe the distribution of M2 macrophages. In HE and Masson staining of liver tissue sections, less fibrosis, granuloma and distribution of M2 macrophages were observed in the liver tissue of mice treated with corilagin intervention than in the model infection, praziquantel or levofloxacin groups. We used praziquantel treatment as a positive control and levofloxacin treatment as a negative control. At 28 days postinfection, all the infected mice were administered praziquantel for 5 days to kill the adult schistosomes. Though the adult schistosomes were killed by praziquantel, the Schistosoma eggs continued to secrete SEA, inducing fibrogenesis. As observed in tissue sections, fibrosis in the praziquantel group and levofloxacin group was much more severe than in the control group and showed no significant difference compared to the model infection group, indicating that the praziquantel has little effect on the progression of fibrogenesis. However, fibrosis was suppressed significantly in the corilagin groups compared with the model infection, praziquantel and levofloxacin groups, demonstrating that corilagin inhibited egg-induced fibrosis more than praziquantel did in vivo.

In this study, we demonstrated the inhibitory effect of corilagin on M2 macrophages and schistosome-egg-induced hepatic fibrosis via IL-13R $\alpha 1$ signaling pathway in vitro and in vivo, especially after the adult schistosomes were killed. Based on the cellular experiment including up- and down-regulation 

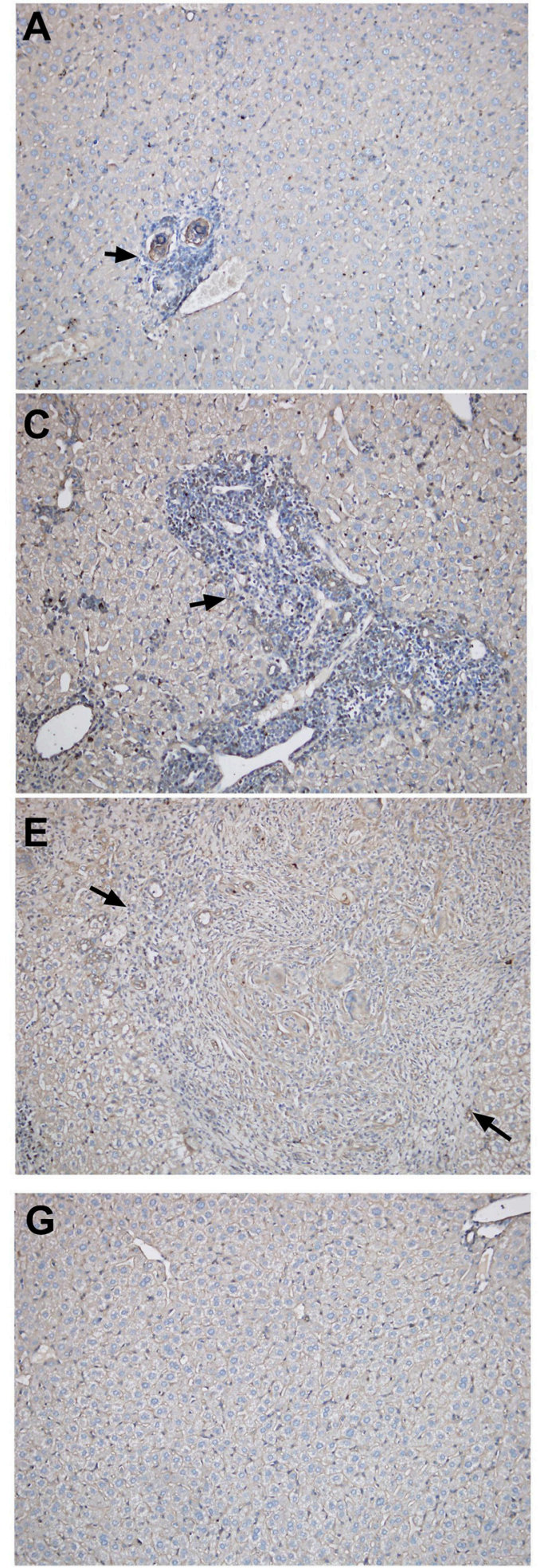
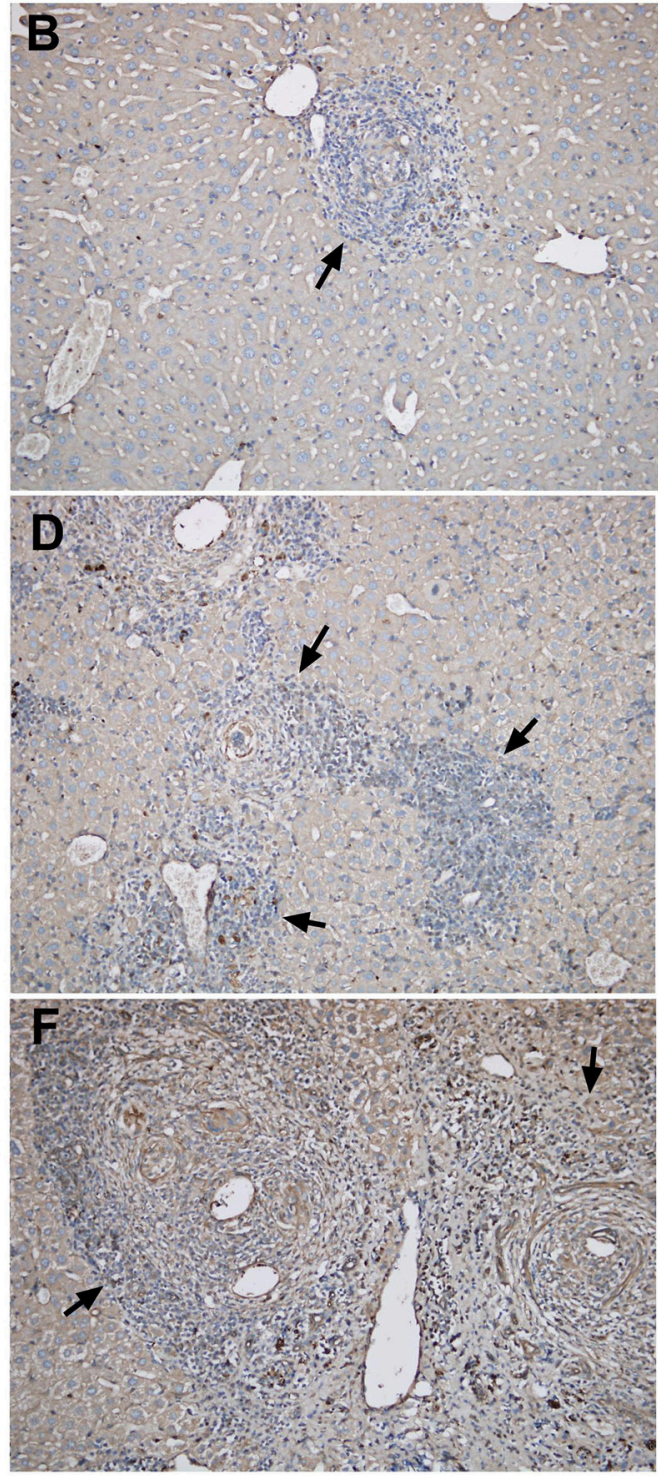

H

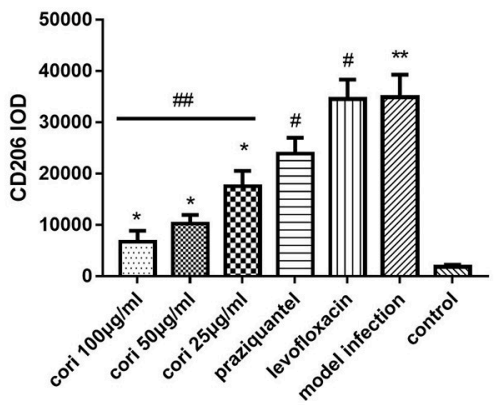

FIGURE 14 | Effect of Corilagin on the distribution of M2 macrophages in animal model examined by immunohistochemistry (200x). After the mice model was established, immunohistochemistry for CD206 was performed. Black arrows indicate the distribution of M2 macrophage. Corilagin treatment significantly reduced the distribution of M2 macrophages in vivo. However, the praziquantel treatment had little effect. (A) High-concentration corilagin group; (B) medium-concentration corilagin group; (C) low-concentration corilagin group; (D) praziquantel group; (E) levofloxacin group; (F) model infection group; (G) control group; (H) CD206 IOD analysis. ${ }^{*} p<0.05$ compared with the model infection group $\left(n=6\right.$, student's $t$-test); ${ }^{*} p<0.01$ compared with the control group $(n=6$, student's $t$-test); $\# p<0.01$ compared with the control group ( $n=6$, student's $t$-test); \#\#p $<0.05$ determined by one-way ANOVA and significant differences from the respective values determined by S-N-K method $(n=6)$. Data are shown with mean with SD. 
of IL-13R $\alpha 1$, we speculated that the results might be attributed to corilagin's interference in the dimerization of IL-13R $\alpha 1$ and IL- $4 \mathrm{R} \alpha$ and (or) the binding of IL-13 to IL-13R $\alpha 1$, leading to decreased affinity of the receptor for IL-13. However, more direct evidence is needed, and we will continue to focus on this aspect. We planned to clarify the further mechanism of corilagin on egg-induced hepatic fibrosis with IL-13 knock-out mice in vivo. Moreover, in view of the crucial role of STAT6 in signal transduction, we will continue to examine this molecule through studies of up- and down-regulation to explore its influence on downstream molecules such as PPAR $\gamma$, KLF4, SOCS1 and TGF$\beta$, and whether STAT6 contributes the anti-fibrogenic effect of corilagin. As currently the effective drug of egg-induced hepatic fibrosis is still relatively lacking, researchers are always trying to find a new drug to treat this disease. In our animal experiments, corilagin more effectively suppressed fibrosis after the adult schistosomes were killed than did praziquantel, which gave us more confidence in this compound that it might provide an extra choice for the patients suffering from this disease and relieve their pain. Based on our experience on the medical effects of natural ingredients (Dang et al., 2017), we believe that corilagin has potential to control schistosome egg-induced hepatic fibrosis in human, bringing new hope to schistosomiasis patients and their family.

\section{REFERENCES}

Anthony, B. J., Ramm, G. A., and McManus, D. P. (2012). Role of resident liver cells in the pathogenesis of schistosomiasis. Trends Parasitol. 28, 572-579. doi: 10.1016/j.pt.2012.09.005

Aron-Wisnewsky, J., Tordjman, J., Poitou, C., Darakhshan, F., Hugol, D., Basdevant, A., et al. (2009). Human adipose tissue macrophages: $\mathrm{ml}$ and $\mathrm{m} 2$ cell surface markers in subcutaneous and omental depots and after weight loss. J. Clin. Endocrinol. Metab. 94, 4619-4623. doi: 10.1210/jc. 2009-0925

Assunção, L. S., Magalhães, K. G., Carneiro, A. B., Molinaro, R., Almeida, P. E., Atella, G. C., et al. (2017). Schistosomal-derived lysophosphatidylcholine triggers M2 polarization of macrophages through PPARgamma dependent mechanisms. Biochim. Biophys. Acta 1862, 246-254. doi: 10.1016/j.bbalip.2016.11.006

Attia, Y. M., Elalkamy, E. F., Hammam, O. A., Mahmoud, S. S., and ElKhatib, A. S. (2013). Telmisartan, an AT1 receptor blocker and a PPAR gamma activator, alleviates liver fibrosis induced experimentally by Schistosoma mansoni infection. Parasit. Vectors 6:199. doi: 10.1186/1756-3305-6-199

Baghy, K., Iozzo, R. V., and Kovalszky, I. (2012). Decorin-TGFbeta axis in hepatic fibrosis and cirrhosis. J. Histochem. Cytochem. 60, 262-268. doi: 10.1369/0022155412438104

Beschin, A., De Baetselier, P., and Van Ginderachter, J. A. (2013). Contribution of myeloid cell subsets to liver fibrosis in parasite infection. J. Pathol. 229, 186-197. doi: $10.1002 /$ path.4112

Biswas, S. K., Chittezhath, M., Shalova, I. N., and Lim, J. Y. (2012). Macrophage polarization and plasticity in health and disease. Immunol. Res. 53, 11-24. doi: 10.1007/s12026-012-8291-9

Biswas, S. K., and Mantovani, A. (2010). Macrophage plasticity and interaction with lymphocyte subsets: cancer as a paradigm. Nat. Immunol. 11, 889-896. doi: $10.1038 /$ ni.1937

Bou Saab, J., Bacchetta, M., and Chanson, M. (2016). Ineffective correction of PPARgamma signaling in cystic fibrosis airway epithelial cells undergoing repair. Int. J. Biochem. Cell Biol. 78, 361-369. doi: 10.1016/j.biocel.2016. 07.035

\section{AUTHOR CONTRIBUTIONS}

Conceived and designed the experiments: LZ. Performed the experiments and analyzed the data: YL, YC, and YD. Contributed reagents/materials/analysis tools and wrote the paper: YL, YC, YD, and LZ. Critical revision of the paper for important intellectual content: YL, YC, YD, YW, ZS, QM, YJW, JZ, LL, QL, and LZ. All authors agree with final approval of the version for submission.

\section{ACKNOWLEDGMENTS}

This work is supported by National Natural Science Foundation of China No. 81371840 and No. 81600373, Hubei Province Health and Family Planning Scientific Research Project No. WJ2017Q021, Hubei Provincial Natural Science Foundation of China No. 2017CFB471, the Fundamental Research Funds for the Central Universities No. 2017KFYXJJ238.

\section{SUPPLEMENTARY MATERIAL}

The Supplementary Material for this article can be found online at: https://www.frontiersin.org/articles/10.3389/fcimb. 2017.00443/full\#supplementary-material

Burke, M. L., Jones, M. K., Gobert, G. N., Li, Y. S., Ellis, M. K., and McManus, D. P. (2009). Immunopathogenesis of human schistosomiasis. Parasite Immunol. 31, 163-176. doi: 10.1111/j.1365-3024.2009.01098.x

Chen, W., Yuan, F., Wang, K., Song, D., and Zhang, W. (2012). Modulatory effects of the acid polysaccharide fraction from one of anamorph of Cordyceps sinensis on Ana-1 cells. J. Ethnopharmacol. 142, 739-745. doi: 10.1016/j.jep.2012.05.055

Chu, D., Du, M., Hu, X., Wu, Q., and Shen, J. (2011). Paeoniflorin attenuates schistosomiasis japonica-associated liver fibrosis through inhibiting alternative activation of macrophages. Parasitology 138, 1259-1271. doi: 10.1017/S0031182011001065

Cioli, D., and Pica-Mattoccia, L. (2003). Praziquantel. Parasitol Res 90(Suppl. 1), S3-S9. doi: 10.1007/s00436-002-0751-z

Colley, D. G., Bustinduy, A. L., Secor, W. E., and King, C. H. (2014). Human schistosomiasis. Lancet 383, 2253-2264. doi: 10.1016/S0140-6736(13)61949-2

Dang, Y. P., Chen, Y. F., Li, Y. Q., and Zhao, L. (2017). Developments of anticoagulants and new agents with anti-coagulant effects in deep vein thrombosis. Mini Rev. Med. Chem. 17, 338-350. doi: 10.2174/1389557516666160609083649

Ding, Y., Li, G., Xiong, L. J., Yin, W., Liu, J., Liu, F., et al. (2015). Profiles of responses of immunological factors to different subtypes of Kawasaki disease. BMC Musculoskelet. Disord. 16:315. doi: 10.1186/s12891-015-0744-6

Dooley, S., and ten Dijke, P. (2012). TGF-beta in progression of liver disease. Cell Tissue Res. 347, 245-256. doi: 10.1007/s00441-011-1246-y

Du, P., Ma, Q., Zhu, Z. D., Li, G., Wang, Y., Li, Q. Q., et al. (2016). Mechanism of Corilagin interference with IL-13/STAT6 signaling pathways in hepatic alternative activation macrophages in schistosomiasisinduced liver fibrosis in mouse model. Eur. J. Pharmacol. 793, 119-126. doi: 10.1016/j.ejphar.2016.11.018

Duan, Y., Gu, X., Zhu, D., Sun, W., Chen, J., Feng, J., et al. (2014). Schistosoma japonicum soluble egg antigens induce apoptosis and inhibit activation of hepatic stellate cells: a possible molecular mechanism. Int. J. Parasitol. 44, 217-224. doi: 10.1016/j.ijpara.2013.11.003

Edwards, J. K. (2015). Renal fibrosis: Activation of JAK3/STAT6 contributes to the development of renal fibrosis. Nat. Rev. Nephrol. 11:445. doi: 10.1038/nrneph.2015.97 
Fabregat, I., Moreno-Càceres, J., Sánchez, A., Dooley, S., Dewidar, B., Giannelli, G., et al. (2016). TGF-beta signalling and liver disease. FEBS J. 283, 2219-2232. doi: $10.1111 /$ febs.13665

Fallon, P. G., Richardson, E. J., McKenzie, G. J., and McKenzie, A. N. (2000). Schistosome infection of transgenic mice defines distinct and contrasting pathogenic roles for IL-4 and IL-13: IL-13 is a profibrotic agent. J. Immunol. 164, 2585-2591. doi: 10.4049/jimmunol.164.5.2585

Gordon, S. (2003). Alternative activation of macrophages. Nat. Rev. Immunol. 3, 23-35. doi: 10.1038/nri978

Gryseels, B., Polman, K., Clerinx, J., and Kestens, L. (2006). Human schistosomiasis. Lancet 368, 1106-1118. doi: 10.1016/S0140-6736(06)69440-3

Guo, Y. J., Luo, T., Wu, F., Liu, H., Li, H. R., Mei, Y. W., et al. (2015a). Corilagin protects against HSV1 encephalitis through inhibiting the TLR2 signaling pathways in vivo and in vitro. Mol. Neurobiol. 52, 1547-1560. doi: 10.1007/s12035-014-8947-7

Guo, Y. J., Luo, T., Wu, F., Mei, Y. W., Peng, J., Liu, H., et al. (2015b). Involvement of TLR2 and TLR9 in the anti-inflammatory effects of chlorogenic acid in HSV-1-infected microglia. Life Sci. 127, 12-18. doi: 10.1016/j.lfs.2015.01.036

Guo, Y. J., Zhao, L., Li, X. F., Mei, Y. W., Zhang, S. L., Tao, J. Y., et al. (2010). Effect of Corilagin on anti-inflammation in HSV-1 encephalitis and HSV-1 infected microglias. Eur. J. Pharmacol. 635, 79-86. doi: 10.1016/j.ejphar.2010.02.049

He, X., Tang, R., Sun, Y., Wang, Y. G., Zhen, K. Y., Zhang, D. M., et al. (2016). MicroR-146 blocks the activation of M1 macrophage by targeting signal transducer and activator of transcription 1 in hepatic schistosomiasis. EBioMedicine 13, 339-347. doi: 10.1016/j.ebiom.2016. 10.024

Herbert, D. R., Hölscher, C., Mohrs, M., Arendse, B., Schwegmann, A., Radwanska, M., et al. (2004). Alternative macrophage activation is essential for survival during schistosomiasis and downmodulates $\mathrm{T}$ helper 1 responses and immunopathology. Immunity 20, 623-635. doi: 10.1016/S1074-7613(04)00107-4

Hesse, M., Modolell, M., La Flamme, A. C., Schito, M., Fuentes, J. M., Cheever, A. W., et al. (2001). Differential regulation of nitric oxide synthase-2 and arginase- 1 by type 1/type 2 cytokines in vivo: granulomatous pathology is shaped by the pattern of L-arginine metabolism. J. Immunol. 167, 6533-6544. doi: 10.4049/jimmunol.167.11.6533

Huang, Y. F., Zhang, S. L., Jin, F., Cheng, D., Zhou, Y. P., Li, H. R., et al. (2013). Activity of corilagin on post-parasiticide liver fibrosis in Schistosomiasis animal model. Int. J. Immunopathol. Pharmacol. 26, 85-92. doi: 10.1177/039463201302600108

Jin, F., Cheng, D., Tao, J. Y., Zhang, S. L., Pang, R., Guo, Y. J., et al. (2013). Antiinflammatory and anti-oxidative effects of corilagin in a rat model of acute cholestasis. BMC Gastroenterol. 13:79. doi: 10.1186/1471-230X-13-79

Jin, F., Zhang, R., Feng, S., Yuan, C. T., Zhang, R. Y., Han, G. K., et al. (2015). Pathological features of transplanted tumor established by CD133 positive TJ905 glioblastoma stem-like cells. Cancer Cell Int. 15:60. doi: 10.1186/s12935-015-0208-y

Kandhi, R., Bobbala, D., Yeganeh, M., Mayhue, M., Menendez, A., and Ilangumaran, S. (2016). Negative regulation of the hepatic fibrogenic response by suppressor of cytokine signaling 1. Cytokine 82, 58-69. doi: 10.1016/j.cyto.2015.12.007

Ke, B., Zhang, A., Wu, X., and Fang, X. (2015). The Role of Kruppel-like Factor 4 in Renal Fibrosis. Front. Physiol. 6:327. doi: 10.3389/fphys.2015.00327

Li, H. R., Li, G., Li, M., Zhang, S. L., Wang, H., Luo, T., et al. (2016). Corilagin ameliorates schistosomiasis hepatic fibrosis through regulating IL13 associated signal pathway in vitro and in vivo. Parasitology 143, 1629-1638. doi: 10.1017/S0031182016001128

Li, H. R., Liu, J., Zhang, S. L., Luo, T., Wu, F., Dong, J. H., et al. (2017). Corilagin ameliorates the extreme inflammatory status in sepsis through TLR4 signaling pathways. BMC Complement. Altern. Med. 17:18. doi: 10.1186/s12906-016-1533-y

Liu, Y., Munker, S., Müllenbach, R., and Weng, H. L. (2012). IL-13 Signaling in liver fibrogenesis. Front. Immunol. 3:116. doi: 10.3389/fimmu.2012.00116

Martinez, F. O., Helming, L., and Gordon, S. (2009). Alternative activation of macrophages: an immunologic functional perspective. Annu. Rev. Immunol. 27, 451-483. doi: 10.1146/annurev.immunol.021908.132532
Pearce, E. J., and MacDonald, A. S. (2002). The immunobiology of schistosomiasis. Nat. Rev. Immunol. 2, 499-511. doi: 10.1038/nri843

Ragheb, S., and Boros, D. L. (1989). Characterization of granuloma T lymphocyte function from Schistosoma mansoni-infected mice. J. Immunol. 142, 3239-3246.

Ross, A. G., Bartley, P. B., Sleigh, A. C., Olds, G. R., Li, Y., Williams, G. M., et al. (2002). Schistosomiasis. N. Engl. J. Med. 346, 1212-1220. doi: 10.1056/NEJMra012396

Sandler, N. G., Mentink-Kane, M. M., Cheever, A. W., and Wynn, T. A. (2003). Global gene expression profiles during acute pathogen-induced pulmonary inflammation reveal divergent roles for Th1 and Th2 responses in tissue repair. J. Immunol. 171, 3655-3667. doi: 10.4049/jimmunol.171.7.3655

Shaker, Y., Samy, N., and Ashour, E. (2014). Hepatobiliary Schistosomiasis. J. Clin. Transl. Hepatol. 2, 212-216. doi: 10.14218/JCTH.2014.00018

Sica, A., and Mantovani, A. (2012). Macrophage plasticity and polarization: in vivo veritas. J. Clin. Invest. 122, 787-795. doi: 10.1172/JCI59643

Song, L. G., and Wu, Z. D. (2015). Pathology and pathogenesis of liver fibrosis induced by Schistosoma japonicum. Zhongguo Xue Xi Chong Bing Fang Zhi Za Zhi 27, 213-216, 220. doi: 10.16250/j.32.1374.2014237

Southgate, V. R., Rollinson, D., Tchuem Tchuenté, L. A., and Hagan, P. (2005). Towards control of schistosomiasis in sub-Saharan Africa. J. Helminthol. 79, 181-185. doi: 10.1079/JOH2005307t

Su, S., Zhao, Q., He, C., Huang, D., Liu, J., Chen, F., et al. (2015). miR-142-5p and miR-130a-3p are regulated by IL-4 and IL-13 and control profibrogenic macrophage program. Nat. Commun. 6:8523. doi: 10.1038/ncomms9523

Wang, T., Huang, W., Costa, M. M., Martin, S. A., and Secombes, C. J. (2011). Two copies of the genes encoding the subunits of putative interleukin (IL)-4/IL-13 receptors, IL-4Ralpha, IL-13Ralpha1 and IL-13Ralpha2, have been identified in rainbow trout (Oncorhynchus mykiss) and have complex patterns of expression and modulation. Immunogenetics 63, 235-253. doi: 10.1007/s00251-0100508-2

Wang, Y., Yang, F., Xue, J., Zhou, X., Luo, L., Ma, Q., et al. (2017). Antischistosomiasis Liver Fibrosis Effects of Chlorogenic Acid through IL13/miR-21/Smad7 Signaling Interactions In Vivo and In Vitro. Antimicrob. Agents Chemother. 61, e01347-16. doi: 10.1128/AAC.01347-16

Wollenberg, A., Mommaas, M., Oppel, T., Schottdorf, E. M., Günther, S., and Moderer, M. (2002). Expression and function of the mannose receptor CD206 on epidermal dendritic cells in inflammatory skin diseases. J. Invest. Dermatol. 118, 327-334. doi: 10.1046/j.0022-202x.2001.01665.x

Xie, Y. Y., Xu, Y. H., Chu, D. Y., and Shen, J. L. (2015). Progress in researches on macrophages in liver fibrosis of schistosomiasis. Zhongguo Xue Xi Chong Bing Fang Zhi Za Zhi 27, 669-673. doi: 10.16250/j.32.1374.2015129

Yang, F., Wang, Y., Xue, J., Ma, Q., Zhang, J., Chen, Y. F., et al. (2016). Effect of Corilagin on the miR-21/smad7/ERK signaling pathway in a schistosomiasis-induced hepatic fibrosis mouse model. Parasitol. Int. 65, 308-315. doi: 10.1016/j.parint.2016.03.001

Yu, J. H., Song, S. J., Kim, A., Choi, Y., Seok, J. W., Kim, H. J., et al. (2016). Suppression of PPARgamma-mediated monoacylglycerol O-acyltransferase 1 expression ameliorates alcoholic hepatic steatosis. Sci. Rep. 6:29352. doi: 10.1038/srep29352

Zhao, L., Zhang, S. L., Tao, J. Y., Pang, R., Jin, F., Guo, Y. J., et al. (2008). Preliminary exploration on anti-inflammatory mechanism of Corilagin (beta-1-O-galloyl-3,6-(R)-hexahydroxydiphenoyl-D-glucose) in vitro. Int. Immunopharmacol. 8, 1059-1064. doi: 10.1016/j.intimp.2008.03.003

Conflict of Interest Statement: The authors declare that the research was conducted in the absence of any commercial or financial relationships that could be construed as a potential conflict of interest.

Copyright (C) 2017 Li, Chen, Dang, Wang, Shang, Ma, Wang, Zhang, Luo, Li and Zhao. This is an open-access article distributed under the terms of the Creative Commons Attribution License (CC BY). The use, distribution or reproduction in other forums is permitted, provided the original author(s) or licensor are credited and that the original publication in this journal is cited, in accordance with accepted academic practice. No use, distribution or reproduction is permitted which does not comply with these terms. 